\title{
Stability and Gradient Dynamical Systems
}

\author{
Jack K. HALE \\ School of Mathematics \\ Georgia Institute of Technology \\ 686 Cherry Street \\ ATLANTA, GA 30332-0160 \\ hale@math.gatech.edu
}

Recibido: 28 de Noviembre de 2003

Aceptado: 17 de Enero de 2004

\begin{abstract}
The objective in these notes is to present an approach to dynamical systems in infinite dimensions. It does not seem reasonable to make a comparison of all of the orbits of the dynamics of two systems on non locally compact infinite dimensional spaces. Therefore, we choose to compare them on the set of globally defined bounded solutions. Fundamental problems are posed and several important results are stated when this set is compact. We then give results on the dynamical system which will ensure that this set is compact. Many applications are give to partial differential equations of parabolic and hyperbolic type as well as functional differential equations.
\end{abstract}

Key words: Dynamical Systems, Stability, Attractors, Gradient systems, Partial differential equations, Functional differential equations

2000 Mathematics Subject Classification: 37Lxx (35B41, 34K25)

If $X$ is a metric space, then any continuous map $T$ that takes $X$ to $X$ is a dynamical system with the dynamics being described by the iterates of $T$. Consider an autonomous evolutionary equation on $X$ for which the initial value problem is well defined. If we define $T(t) x, t \geq 0$, as the solution through $x$, then the family of mappings $\{T(t), t \geq 0\}$ is a dynamical system on $X$.

For the case in which $X$ is a compact manifold (or even locally compact), there is an extensive qualitative theory of dynamical systems associated with the stability and bifurcation of the orbit structure. 
If $X$ is not locally compact, the theory is far from complete. From investigations of functional differential equations which began in the late 1950's, an abstract framework evolved and an important class of dynamical systems were identified for which a qualitative theory is possible in the nonlocally compact case. The abstract theory applies also to many classes of partial differential equations including quasilinear parabolic equations and many types of hyperbolic equations. For this reason, we include in the appendix some of the basic properties of functional differential equations of retarded and neutral type.

One first objective in these notes is to introduce these ideas and to state a few of the important results that have been obtained. After a general discussion of gradient systems, we present examples from functional differential equations, quasilinear parabolic equations and linearly damped nonlinear wave equations.

The hope is that the interested reader will consult the many references given in the text for a more extensive view of the subject.

\section{Basic concepts}

Suppose that $X$ is a metric space with metric $d_{X}$. Let $\mathbf{Z}=\{0, \pm 1, \pm 2, \ldots\}, \mathbf{Z}^{+}=$ $\{0,1,2, \ldots\}, \mathbf{Z}^{-}=\{0,-1,-2, \ldots\}$. A discrete dynamical system is the family of mapping $\left\{T^{n}, n \in \mathbf{Z}^{+}\right\}$where $T: X \rightarrow X$ is a continuous map.

Let $\mathbb{R}=(-\infty, \infty), \mathbb{R}^{+}=[0, \infty), \mathbb{R}^{-}=(-\infty, 0]$. A mapping $T: \mathbb{R}^{+} \times X \rightarrow X$, $(t, x) \mapsto T(t) x$ is said to be a $C^{0}$-semigroup (or a continuous dynamical system) (or a $C^{0}$-dynamical system) if

(i) $T(0)=I$,

(ii) $T(t+s)=T(t) T(s), t, s \in \mathbb{R}^{+}$,

(iii) $T(t) \in C^{0}(X, X)$ for $t \geq 0$,

(iv) $T(t) x$ is continuous in $t, x$ for $(t, x) \in[0, \infty) \times X$.

In some applications, it is necessary to consider the case where $T(t) x$ is only continuous for $(t, x) \in(0, \infty) \times X$. To handle this case requires only a few changes which are not discussed..

It is convenient to consider the discrete dynamical system and the $C^{0}$-dynamical system together by letting $\mathbf{G}$ be either $\mathbb{R}$ or $\mathbf{Z}$ with similar definitions for $\mathbf{G}^{+}$and $\mathbf{G}^{-}$and write the dynamical system as $T: \mathbf{G}^{+} \times X \rightarrow X$.

For any $x \in X$, the positive orbit $\gamma^{+}(x)$ through $x$ is defined as $\gamma^{+}(x)=$ $\bigcup_{t \in \mathbf{G}^{+}} T(t) x$. A continuous function $y: \mathbf{G}^{-} \rightarrow X$ defines a negative orbit $\gamma^{-}(x)$ through $x$ if, for any $t, s \in \mathbf{G}^{-}, s \geq t, T(s-t) y(t)=y(s)$. A complete orbit $\gamma(x)$ through $x$ is the union of $\gamma^{+}(x)$ and a negative orbit through $x$.

Since the range of $T(t)$ need not be the whole space, to say that there is a negative orbit through $x$ may impose restrictions on $x$. Since $T(t)$ may not be one-to-one, there 
may be more than one negative orbit through $x$ if one exists. We define the negative orbit $\Gamma^{-}(x)$ through $x$ as the union of all negative orbits through $x$. The complete orbit $\Gamma(x)$ through $x$ is $\Gamma(x)=\gamma^{+}(x) \cup \Gamma^{-}(x)$.

For any subset $B$ of $X$, we let $\gamma^{+}(B)=\bigcup_{x \in B} \gamma^{+}(x), \Gamma^{-}(B)=\bigcup_{x \in B} \Gamma^{-}(x)$, $\Gamma(B)=\bigcup_{x \in B} \Gamma(x)$ be respectively the positive orbit, negative orbit, complete orbit through $B$.

The orbit structure or flow defined by a dynamical system is the set of all positive, negative and complete orbits together with a designation of the direction of the movement along an orbit with increasing $t \in \mathbf{G}^{+}$.

A set $J \subset X$ is an invariant set of the dynamical system $T$ if $T(t) J=J$ for $t \in \mathbf{G}^{+}$. If $J$ is invariant and $x \in J$, then there must be a complete orbit $\gamma(x)$ through $x$ which we denote by $\{T(t) x, t \in \mathbf{G}\}$.

We also need the concept of positive limit and negative limit of points and sets. For $x \in X$, we define $\omega(x)$, the $\omega$-limit set of $x$ or the $\omega$-limit set of the orbit through $x$, as

$$
\omega(x)=\bigcap_{\tau \in \mathbf{G}+} \mathrm{Cl} \gamma^{+}(T(\tau) x),
$$

where ' $\mathrm{Cl}$ ' denotes closure in $X$. This is equivalent to saying that $y \in \omega(x)$ if and only if there is is a sequence $t_{k} \in \mathbf{G}^{+}, t_{k} \rightarrow \infty$ as $k \rightarrow \infty$ such that $T\left(t_{k}\right) x \rightarrow y$ as $k \rightarrow \infty$. In the same way, for any set $B \subset X$, we define $\omega(B)$, the $\omega$-limit set of $B$ or the $\omega$-limit set of the orbit through $B$, as

$$
\omega(B)=\bigcap_{\tau \in \mathbf{G}^{+}} \mathrm{Cl} \gamma^{+}(T(\tau) B) .
$$

This is equivalent to saying that $y \in \omega(B)$ if and only if there are sequences $t_{k} \in \mathbf{G}^{+}, t_{k} \rightarrow \infty$ as $k \rightarrow \infty, x_{k} \in B$, such that $T\left(t_{k}\right) x_{k} \rightarrow y$ as $k \rightarrow \infty$.

We remark that $\omega(B)$ may not be the union of $\omega(x)$ for $x \in B$.

In the same way, we can define the $\alpha$-limit set of a negative orbit $\gamma^{-}(x)$ or the negative orbit $\Gamma^{-}(x)$ of a point $x$ as well as the same concept for a set $B \subset X$.

Note that $\omega$-limit sets and $\alpha$-limit sets are invariant sets.

Our exposition below will require other concepts which we now introduce. A set $A \subset X$ attracts a set $B \subset X$ under the dynamical system $T$ if

$$
\lim _{t \rightarrow \infty} \operatorname{dist}_{X}(T(t) B, A)=0,
$$

where

$$
\operatorname{dist}_{X}(B, A) \equiv \sup _{x \in B} \operatorname{dist}_{X}(x, A) \equiv \sup _{x \in B} \inf _{y \in A} \operatorname{dist}_{X}(x, y) .
$$

We say that a dynamical system $T$ is point dissipative if there is a bounded set $B \subset X$ which attracts each point of $X$. This concept was introduced by Levinson (1944) for $X=\mathbb{R}^{n}$ in his study of the periodically forced van der Pol equation. 
This dissipative condition avoids the discussion of the detailed properties of the orbit structure for large values of $x$.

For a given dynamical system $T$, we let

$$
\mathcal{A}(T)=\{x \in X: T(t) x \text { is defined and bounded for } t \in \mathbf{G}\} .
$$

be the set of bounded global orbits of $T$. The set $\mathcal{A}(T)$ is invariant.

If $\mathcal{A}(T)$ is compact, it is the maximal compact invariant set of $T$; that is, $\mathcal{A}(T)$ is compact, invariant and maximal with respect to this property. We remark that, if $X$ is connected, then the maximal compact invariant set is connected if $\mathbf{G}=\mathbb{R}$ and is invariantly connected if $\mathbf{G}=\mathbf{Z}$. A set $J \subset X$ is invariantly connected with respect to a map $T$ if it cannot be represented as $J=J_{1} \cup J_{2}$ with $J_{1}, J_{2}$ being nonempty, disjoint, closed sets satisfying $T J_{j} \subset J_{j}, j=1,2$ (LaSalle (1976)).

We say that a set $A \subset X$ attracts a set $B \subset X$ under the action of the dynamical system $T: \mathbf{G}^{+} \times X \rightarrow X$ if $\operatorname{dist}_{X}(T(t) B, A) \rightarrow 0$ as $t \rightarrow \infty$. The set $\mathcal{A}(T)$ is the compact global attractor of $T$ if it is compact, invariant and attracts each bounded set of $X$.

\section{Stability for $X$ compact}

There is an extensive theory for flows defined by dynamical systems when $X$ is either a finite dimensional compact manifold without boundary or a compact manifold with boundary provided that the flow is differentiable and transversal to the boundary. The basic problem is to compare the flows defined by different dynamical systems. The comparison in these cases is made most often through the notion of topological equivalence. Two dynamical systems $T$ and $S$ defined on $X$ are topologically equivalent, $T \sim S$, if there is a homeomorphism from $X$ to $X$ which takes the orbits of $T$ onto the orbits of $S$ and preserves the sense of direction in time.

Dynamical systems defined from models in applications always involve parameters and it is necessary to understand when the orbit structure does not change under perturbations of the parameters and when it does. Let us make this more precise.

Suppose that $X$ is a compact metric space, $\Lambda$ is a metric space, $T: \Lambda \times \mathbf{G}^{+} \times$ $X \rightarrow X$ is continuous and, for each $\lambda \in \Lambda$, let $T_{\lambda}: \mathbf{G}^{+} \times X \rightarrow X$ be defined by $T_{\lambda}(t) x=T(\lambda, t, x)$. Also, suppose that, for each $\lambda \in \Lambda, T_{\lambda}$ is a dynamical system on $X$ with the property that, for each $x \in \partial X, t>0, T_{\lambda}(t) x$ is in the interior of $X$. The dynamical system $T_{\lambda_{0}}, \lambda_{0} \in \Lambda$ fixed, is said to be stable if there is a neighborhood $U \subset \Lambda$ of $\lambda_{0}$ such that $T_{\lambda_{0}} \sim T_{\lambda}$ for each $\lambda \in U$. We say that $T_{\lambda_{0}}$ is a bifurcation point if $T_{\lambda_{0}}$ is not stable.

For $X$ either a smooth compact manifold without boundary or one with boundary for which the flow is transversal to the boundary, much research has been devoted to the characterization of those dynamical systems which are stable (see, for example, Palis and de Melo (1982)). A complete characterization of stable dynamical systems generated by ordinary differential equations in the plane was given by Andronov and 
Pontryagin (1937) with the parameter space being the $C^{1}$-vector fields on $X$. Several types of bifurcation points also have been analyzed completely. A good historical perspective on these topics is contained in Aubin and Dahan Dalmedico (2002).

\section{Stability for $X$ locally compact}

If the dynamical system is defined on a finite dimensional Banach space $X$, then extreme care must be exercised in order to compare the orbits with large initial data and only very special cases have been considered. One way to avoid the consideration of large initial data in the comparison of dynamical systems is to consider only those for which infinity is unstable. The precise concept is point dissipativeness which we have defined in Section 1. The following result is essentially due to Pliss (1966).

Theorem 3.1. If $T: \mathbf{G}^{+} \times X \rightarrow X$ is a dynamical system on a locally compact metric space $X$ which is point dissipative, then the compact global attractor $\mathcal{A}(T)$ exists.

The proof relies heavily upon the fact that the closure of any bounded set in $X$ is compact. From this fact, one can show that, for any bounded set $U \subset X, \gamma^{+}(U)$ is bounded and has compact closure. Therefore, $\omega(U)$ is a compact invariant set. The attractor can be shown to be $\omega(B)$ where $B$ is the bounded set in the definition of point dissipativeness.

Under the conditions of Theorem 3.1, if $X$ is a finite dimensional Banach space, then it is often possible to find a neighborhood $\mathcal{M}$ of $\mathcal{A}(T)$ for which the closure is a compact manifold with boundary and the flow is transversal to the boundary of $\mathcal{M}$. Therefore, the above mentioned global theory of finite dimensional dynamical systems can be applied.

\section{Stability for general $X$}

Topological dynamics deals with dynamical systems generated by transformation groups $T(t), t \in \mathbb{R}$, on metric spaces $X$. There is an extensive theory which does not take into account any special properties of the transformations other than continuity properties of $T(t) x$ or differentiability properties of this function with respect to $x$ (see, for example, Gottschalk and Hedlund (1955), Bhatia and Szegö (1967) for an introduction). This implies that most of the results will be valid only for locally compact spaces $X$. Thus, if $X$ is not locally compact, this limits the applicability of the theory. For example, if $J$ is a invariant set, we may define stability of $J$ in the sense of Lyapunov to mean that, for any neighborhood $U$ of $J$, there is a neighborhood $W$ of $J$ such that, $T(t) W \subset U$ for $t \geq 0$. On the other hand, the strongest meaningful definition of $J$ being a local attractor would be that there is a neighborhood $V$ of $J$ such that, for any compact set $K \subset V$, the set $J$ attracts $K$. We would not 
expect in the general situation that $J$ is a local attractor in the sense that $J$ attracts a neighborhood of itself.

It is clear that it is desirable to obtain conditions on the dynamical system which will imply the stronger attractivity property and, at the same time, be of interest in the applications. To accomplish this, one must take advantage of additional properties of the dynamical system. A large class of dynamical systems have been identified as a result of a detailed study of dynamical systems generated by partial differential equations and delay differential or functional differential equations. (see Henry (1981), (1987), Hale (1977), (1987), Ladyženskaja (1991), Babin and Vishik (1992), Hale and Verduyn Lunel (1993), Diekmann et al. (1995), Temam (1997), Sell and You (2002), Raugel (2002), Hale et al. (2002) and the references therein). In spite of this fact, the theory is far from being developed.

Following Hale et al. (2002), we outline the manner in which it is possible to begin to develop the theory by an appropriate modification of ideas and methods used in the finite dimensional theory. The set $\mathcal{A}(T)$ plays a central role.

If $X$ is a not a locally compact metric space and $T$ and $S$ are dynamical systems on $X$, then it seems to be almost impossible to make a comparison of all or even an arbitrary bounded set of orbits of the space $X$. For this reason, we make comparisons of dynamical systems only on $\mathcal{A}(T)$. This does not mean that the transient behavior is unimportant, but only that our emphasis here is on $\mathcal{A}(T)$. The following definition first appeared in Hale (1981).

Definition 4.1. We say that a dynamical system $T$ on $X$ is equivalent to a dynamical system $S$ on $X, T \sim S$, if there is a homeomorphism $h: \mathcal{A}(T) \rightarrow \mathcal{A}(S)$ which preserves orbits and the sense of direction in time.

We reemphasize that, in the definition of equivalence, we restrict to the set $\mathcal{A}(T)$ and not to a neighborhood of $\mathcal{A}(T)$. Due to the fact that we are not able to take this full neighborhood, adaptation of the finite dimension theory of dynamical systems to this infinite dimensional setting is nontrivial. Also, further restrictions must be imposed on the classes of dynamical systems that will be considered.

As remarked earlier, in applications, the dynamical system depends upon parameters and it is necessary to understand the changes in the orbit structure when subjected to variations in these parameters. A particular physical problem is modeled by a dynamical system containing only enough parameters to reflect the experimental observations made on the system. In the case of ordinary differential equations or functional differential equations, the parameter could be a particular class of vector fields. If the dynamical system is generated by a partial differential equations, the parameters could be a class of vector fields or the boundary of the region of definition or the boundary conditions or all of these.

A basic problem is to know if the flow defined by a dynamical system is preserved under the above equivalence relation when one allows variations in the parameters. More precisely, we make the following definitions which are natural generalizations of 
the case when $X$ is compact except that we restrict to the set of bounded globally defined orbits.

Definition 4.2. Suppose that $X$ is a complete metric space, $\Lambda$ is a metric space, $T: \Lambda \times \mathbf{G}^{+} \times X \rightarrow X$ is continuous and, for each $\lambda \in \Lambda$, let $T_{\lambda}: \mathbf{G}^{+} \times X \rightarrow X$ be defined by $T_{\lambda}(t) x=T(\lambda, t, x)$ and suppose that $T_{\lambda}$ is a dynamical system on $X$ for each $\lambda \in \Lambda$. Define $\mathcal{A}\left(T_{\lambda}\right)$ as above. The dynamical system $T_{\lambda}$ is said to be $\mathcal{A}$-stable if there is a neighborhood $U \subset \Lambda$ of $\lambda$ such that $T_{\lambda} \sim T_{\mu}$ for each $\mu \in U$. We say that $T_{\lambda}$ is a bifurcation point if $T_{\lambda}$ is not $\mathcal{A}$-stable.

In studying the stability of the flow of a given dynamical system $T$, it is probably necessary to know that the flow is one-to-one on $\mathcal{A}(T)$ and, thus, the question:

$\mathbf{Q}$ 4.1. Under what conditions on a dynamical system $T$ is the flow one-to-one on $\mathcal{A}(T)$ ?

For general classes of quasilinear parabolic partial differential equations on a bounded domain, the corresponding continuous dynamical system on $\mathbb{R}^{+}$is one-to-one on all of $X$.

For other classes of evolutionary equations, such a strong result is not to be expected for every $x \in X$. On the other hand, there is the possibility that there are large classes of evolutionary equations for which one obtains one-to-oneness on compact invariant sets.

If a continuous dynamical system on $\mathbb{R}^{+}$has the property that, for each compact invariant set $J \subset X$ and for each $x \in J$, the function $\{T(t) x, t \in \mathbb{R}\}$ is analytic in $t$, then $T$ is one-to-one on $J$.

For retarded functional differential equations with finite delay for which the vector field is analytic, it was shown by Nussbaum (1973) that, for any compact invariant set $J$ and any $x \in J$ the function $T(t) x$ defining a complete orbit in $J$ is analytic in $t \in \mathbb{R}$. We remark that, even though the vector field is analytic, the function $T(t) x$ is not analytic for each $x \in X$.

There are examples of retarded functional differential equations for which the vector field is $C^{\infty}$ and one does not have one-to-oneness on a compact invariant set (see, for example, Hale et al. (2002)).

Hale and Scheurle (1985) obtained the same analyticity result for neutral functional differential equations with an exponentially stable $D$ operator, a class of equations which includes the retarded case.

If $J$ is a compact invariant set for a continuous dynamical system $T: \mathbb{R}^{+} \times X \rightarrow X$ generated by abstract evolutionary equations, other results on analyticity of $T(t) x$ in $t \in \mathbb{R}$ are in Hale and Raugel (2003). The equations include many classes of dissipative hyperbolic equations. This paper also contains results on $C^{k}$ regularity in $t$. We remark also that these regularity properties in $t$ often imply regularity in the spatial variables for the case when the evolutionary equation corresponds to a partial differential equation. 
For a parametrized family of dynamical systems as in Definition 4.2, it is important to investigate the following questions.

Q 4.2. Generically in $\Lambda$, is $T_{\lambda}$ one-to-one on $\mathcal{A}\left(T_{\lambda}\right)$ ?

Q 4.3. If $T_{\lambda}$ is $\mathcal{A}$-stable, is $T_{\lambda}$ one-to-one on $\mathcal{A}\left(T_{\lambda}\right)$ ?

Sternberg (1990) has given a positive answer to these questions for retarded functional differential equations with finite delay on a compact manifold without boundary. The parameter space $\Lambda$ is the class of $C^{1}$-vector fields.

$\mathrm{Q}$ 4.4. Is the Hausdorff dimension of $\mathcal{A}(T)$ finite? Is the capacity (box dimension) of $\mathcal{A}(T)$ finite?

If $\mathcal{A}(T)$ is compact and $X$ is a Hilbert space, Mallet-Paret (1976) showed that the Hausdorff dimension of $\mathcal{A}(T)$ is finite if $T(t)=S(t)+U(t)$, where $U(t)$ is a compact map for each $t \in \mathbf{G}^{+}$and $S(t)$ is a linear operator with the property that $\|S(t)\| \rightarrow 0$ as $t \rightarrow \infty$ in G. Mañé (1981) proved a more general result for $X$ a Banach space. More specifically, he proved the capacity of $\mathcal{A}(T)$ is finite under the hypothesis that $T$ has the above decomposition. He showed also that, in the class of linear subspaces of dimension $>2 c(A)+1$, there is a residual set $\mathcal{U}$ such that, for each $S \in \mathcal{U}$, the projection of $\mathcal{A}(T)$ onto $S$ is one-to-one. For a complete proof, see Hale et al. (2002).

Retarded and large classes of neutral functional differential equations as well a many dissipative parabolic and hyperbolic equations generate dynamical systems for which the above decomposition holds. (see, for example, Hale (1987), Hale et al. (2002), Temam (1997)). For estimates of this capacity in specific systems, see, for example, Temam (1997).

Q 4.5. When is $\mathcal{A}(T)$ a manifold or the union of a finite number of manifolds?

We will see below that, if the dynamical system is gradient with all equilibria hyperbolic and $\mathcal{A}(T)$ compact, then $\mathcal{A}(T)$ is the union of a finite number of manifolds.

In the case where $X$ is a compact manifold, Morse-Smale systems have played a very important role. The same is true in infinite dimensions. To define a Morse-Smale system, we need some remarks about hyperbolicity of equilibria and periodic orbits.

Definition 4.3. A point $x_{0} \in X$ is an equilibrium (or critical point) of the flow for a dynamical system $T$ if $T(t) x_{0}=x_{0}$ for all $t \in \mathbf{G}^{+}$. An orbit $\Gamma$ is a periodic orbit of least period $\tau$ if $\Gamma=\{p(t), t \in \mathbf{G}\}$ where $p(t+\tau)=p(t)$ for $t \in \mathbf{G}^{+}$and $p(t+s) \neq p(t)$ for any $s \in(0, \tau) \cap \mathbf{G}^{+}$.

If the dynamical system is discrete and defined by a continuous mapping $T$ : $X \rightarrow X$, then an equilibrium (or critical point) is a fixed point of $T$. A periodic orbit $p$ of minimal period $\tau$ ( $\tau \geq 1$ must be an integer) is a sequence of $\tau$ distinct points $p=\left\{x_{0}, x_{1}, \ldots, x_{\tau-1}\right\}$ with the property that $x_{j}=T^{j} x_{0}, 0 \leq j \leq \tau-1$, and $T^{\tau} x_{0}=x_{0}$. 
For a continuous dynamical system $T$ on $\mathbb{R}^{+}$, an equilibrium is a point $x_{0}$ such that $T(t) x_{0}=x_{0}$ for each $t \geq 0$. A periodic orbit of minimal period $\tau>0$ is a closed curve in $X$.

If $x_{0}$ is an equilibrium, then one can define the stable set $W^{s}\left(x_{0}\right)$ (resp. the unstable set $\left.W^{u}\left(x_{0}\right)\right)$ of $x_{0}$ as the set of all initial values $x \in X$ such that $T(t) x \rightarrow x_{0}$ as $t \rightarrow \infty$ (resp. $t \rightarrow-\infty$ ). If $T(t) x$ is $C^{1}$ in $X$, then we can define $D T(1)\left(x_{0}\right)$ as the linear operator given by the derivative of $T(t) x$ with respect to $x$ evaluated at $t=1, x=x_{0}$. The point $x_{0}$ is hyperbolic if the spectrum of $D T(1)\left(x_{0}\right)$ is bounded away from the unit circle with center zero in the complex plane. If $x_{0}$ is hyperbolic, then there is a neighborhood $U$ of $x_{0}$ such that $W^{s}\left(x_{0}\right) \cap U$ (resp. $\left.W^{u}\left(x_{0}\right) \cap U\right)$ is a manifold which is $C^{k}$ if $T(t) x$ is $C^{k}$ in $x$. If $T$ is one-to-one, then $W^{s}\left(x_{0}\right)$ (resp. $\left.W^{u}\left(x_{0}\right)\right)$ is a manifold.

For a discrete dynamical system defined by a $C^{k}$-map $T: X \rightarrow X, k \geq 1$, one can define hyperbolicity of a periodic orbit of minimal period $\tau$ in the same way as above by using the spectrum of the derivative $D T^{\tau}\left(x_{0}\right)$.

For a continuous dynamical system $T: \mathbb{R}^{+} \times X \rightarrow X$, let $\Gamma=\{p(t), t \in \mathbb{R}\}$, $p(t)=T(t) p(0), t \in \mathbb{R}$, be a periodic orbit of minimal period $\tau$. As for an equilibrium, we define

$$
\begin{aligned}
& W^{s}(\Gamma)=\left\{x \in X: \lim _{t \rightarrow \infty} \operatorname{dist}_{X}(T(t) x, \Gamma)=0\right\} \\
& W^{u}(\Gamma)=\left\{x \in X: \lim _{t \rightarrow-\infty} \operatorname{dist}_{X}(T(t) x, \Gamma)=0\right\}
\end{aligned}
$$

as the stable and unstable sets of $\Gamma$.

To define hyperbolicity of $\Gamma$, it is necessary to discuss some of the properties of $W^{s}(\Gamma)$ and $W^{u}(\Gamma)$ in a neighborhood of $\Gamma$. The usual way of accomplishing this is the following. If $\Gamma$ is a $C^{1}$-manifold, then one first chooses a transversal $\Sigma$ to the curve $\Gamma$ at some point, say $p_{0}=p(0)$. Then there is a neighborhood $U$ in $\Sigma$ of $p_{0}$, such that, for any $x \in U$, there is a continuous function $\alpha(x), \alpha\left(p_{0}\right)=\tau$ such that $\tilde{T} x \equiv T(\alpha(x))(x) \in \Sigma$. This map is called the Poincaré map on the transversal $\Sigma$. If $\tilde{T}(x)$ is a $C^{1}$-function, then we can define hyperbolicity of the fixed point $p_{0}$ of $\tilde{T}(x)$ in the same way as for an equilibrium by requiring that the spectrum of $D \tilde{T}\left(p_{0}\right)$ has no element of modulus one. Knowing the stable and unstable manifolds in $\Sigma$ for the fixed point $p_{0}$, one obtains information about $W^{s}(\Gamma)$ and $W^{u}(\Gamma)$.

If the space $X$ is a finite dimensional smooth manifold, then the map $\tilde{T}(x)$ is a $C^{1}$-function and the above procedure works very well.

If the dynamical system corresponds to either a parabolic partial differential equation or a retarded functional differential equation with finite retardation, then the differentiability properties of $\tilde{T}(x)$ also are satisfied for some multiple of the period. Therefore, the Poincaré map can be used to determine the properties of the flow near $\Gamma$.

For the case of general dynamical systems on an infinite dimensional space $X$, two problems arise in trying to use the Poincaré map on a transversal to $\Gamma$. One 
must first be able to define a transversal which implies that we must know that $\Gamma$ is a $C^{1}$-manifold. If $\Gamma$ is a $C^{1}$-manifold, then we must have the Poincaré map to be $C^{1}$. For many evolutionary equations corresponding to interesting classes of neutral functional differential equations and dissipative hyperbolic partial differential equations, a periodic orbit $\Gamma$ is a $C^{1}$-manifold and, thus, it is possible to define a transversal to $\Gamma$ and to define the Poincaré map. However, it is usually not the case in these situations that the Poincaré map is a $C^{1}$-function. Therefore, one must define hyperbolicity and obtain regularity properties of the stable and unstable sets in another way.

If $\Gamma=\{p(t), t \in \mathbb{R}\}$ is a periodic solution of an evolutionary equation, then we can define the linear variational equation about $p(t)$. This will be a linear evolutionary equation with periodic coefficients of period $\tau$. Define $S$ to be the mapping which takes the initial value $x_{0}$ at time zero to the solution through $x_{0}$ at time $\tau$. If $\Gamma$ is a $C^{1}$-manifold, then $\dot{p}(t)$ is a periodic solution of the linearly variational equation and, therefore, 1 belongs to the spectrum $\sigma(S)$ of $S$. We say that $\Gamma$ is hyperbolic if 1 is a simple eigenvalue of $S$ and no element of $\sigma(S) \backslash 1$ has modulus one. With this definition, one can show, in many situations, that hyperbolicity implies that $W^{s}(\Gamma)$ and $W^{u}(\Gamma)$ are $C^{1}$ manifolds.

This is sufficient to motivate the following definition.

Definition 4.4. A dynamical system $T: \mathbf{G}^{+} \times X \rightarrow X$ is Morse-Smale if

(i) there are only a finite number of equilibria, each is hyperbolic with smooth stable and unstable manifolds,

(ii) there are only a finite number of periodic orbits, each is hyperbolic with smooth stable and unstable manifolds,

(iii) stable and unstable manifolds of equilibria and periodic orbits intersect transversally,

(iv) the union of the equilibria and periodic orbits coincides with the nonwandering set $\Omega(T)$.

For a dynamical system $T$ on $\mathbf{G}^{+} \times X$, the nonwandering set $\Omega(T)$ is defined as follows:

$$
\begin{aligned}
\Omega(T)=\{x \in X: \forall \text { neighborhood } V \text { of } x & , \forall t_{0} \\
\qquad & \in \mathbf{G}^{+}, \\
& \left.\exists t \in \mathbf{G}^{+}, t>t_{0}, \text { with } T(t) V \cap V \neq \emptyset\right\}
\end{aligned}
$$

For parametrized dynamical systems as in Definition 4.2, it is natural to ask if Morse-Smale systems are $\mathcal{A}$-stable.

If $X$ is a compact manifold, a Morse-Smale system is stable in the sense of Section 2 in the class of $C^{1}$-perturbations (see, for example, Smale (1967)). Oliva (see Hale et al. (2002) for precise references) has given a class of dynamical systems in the infinite 
dimensional case for which Morse-Smale systems are $\mathcal{A}$-stable. To avoid introducing further notation, we state a special case of his result for maps and some types of evolutionary equations.

Theorem 4.5. Consider a parameterized family of dynamical systems as in Definition 4.2. For each $\lambda \in \Lambda$, assume that $T_{\lambda}(t) x$ is $C^{1}$ in $X$ and one-to-one together with its first derivative in $x$. For a fixed $\lambda_{0} \in \Lambda$, suppose that there is a neighborhood $U$ of $\lambda_{0}$ such that, for any $\lambda \in U$, the set $\mathcal{A}\left(T_{\lambda}\right)$ is compact and the family of sets $\left\{\mathcal{A}\left(T_{\lambda}\right), \lambda \in U\right\}$ is upper semicontinuous at $\lambda_{0}$; that is, $\operatorname{dist}_{X}\left(\mathcal{A}\left(T_{\lambda}\right), \mathcal{A}\left(T_{\lambda_{0}}\right)\right) \rightarrow 0$ as $\lambda \rightarrow \lambda_{0}$.

The following conclusions hold:

(i) For discrete dynamical systems, if $T_{\lambda_{0}}$ is Morse-Smale, then it is $\mathcal{A}$-stable.

(ii) If the dynamical system $T_{\lambda}$ corresponds either to a retarded functional differential equation with finite delay or a parabolic system of partial differential equations on a bounded domain and $T_{\lambda_{0}}$ is Morse-Smale, then $T_{\lambda_{0}}$ is $\mathcal{A}$-stable.

In the more general result of Oliva, some regularity properties in $t$ are required which are not satisfied for neutral functional differential equations with finite delay, retarded equations with infinite delay, as well as systems of partial differential equations which do not have smoothing properties in $t$. An important problem is to extend the result so as to apply to these situations. One should be able to exploit the special properties of the dynamical systems associated with these equations. In the discussion in the next section we mention these properties. Many specific situations satisfying these properties are contained in the general references given at the beginning of Section 4 .

\section{Attractivity properties for general $X$}

In nonlocally compact spaces, we have noted the role played by the set $\mathcal{A}(T)$ in $(1)$ for the qualitative theory of dynamical systems and some of the advantages of having this set compact. In this section, we identify those dynamical systems for which $\mathcal{A}(T)$ is compact.

In Section 3, for $X$ locally compact, we have noted that, if the dynamical system is point dissipative, then $\mathcal{A}(T)$ is the compact global attractor. If the dynamical system satisfies some additional properties, then the same result is valid even if $X$ is not locally compact. In fact, Billotti and LaSalle (1971) proved the following result.

Theorem 5.1. If $T$ is a dynamical system on a metric space $X$ for which there is a $t_{1}>0$ such that $T\left(t_{1}\right)$ is completely continuous and $T$ is point dissipative, then the compact global attractor exists.

This result is very useful for retarded functional differential equations with finite delay and parabolic equations. 
If $X$ is not locally compact, then it is not to be expected that such a strong result would be implied unless $T(t)$ became smooth after some finite $t_{1}>0$. In fact, this is demonstrated by the following simple result in Hale (2000).

Theorem 5.2. Suppose that $X$ is a Banach space and $T: \mathbf{G}^{+} \times X \rightarrow X$ is a dynamical system on $X$ for which $T(t): X \rightarrow X$ is linear for each $t \in \mathbf{G}^{+}$. Let $r\left(\sigma_{\text {ess }}(T(1))\right)$ be the essential spectral radius of $T(1)$. If $T$ is point dissipative (equivalently the origin attracts points of $X)$, then

(i) $r\left(\sigma_{\text {ess }}(T(1))\right) \leq 1$,

(ii) if $r\left(\sigma_{\text {ess }}(T(1))\right)=1$, then $\mathcal{A}(T)=\{0\}$ is the maximal compact invariant set, $\mathcal{A}(T)$ attracts compact sets of $X, \gamma^{+}(B)$ is bounded if $B$ is bounded, but $\mathcal{A}(T)$ does not attract bounded sets of $X$,

(iii) if $r\left(\sigma_{\text {ess }}(T(1))\right)<1$, then $\mathcal{A}(T)=\{0\}$ is the compact global attractor.

It is instructive to say more about the differences between (ii) and (iii) in Theorem 5.2. If $T$ is a linear dynamical system on a Banach space $X$, then there is an equivalent norm in $X$ such that $T(1)$ can always be expressed as

$$
T(1)=S+U
$$

where $U$ is a compact linear operator and $\|S\|=r\left(\sigma_{\text {ess }}(T(1))\right)$ (see, for example, Deimling (1985)). If $r\left(\sigma_{\text {ess }}(T(1))\right)<1$ and $T$ is point dissipative, then this representation shows that the approach to zero of a solution is exponential and the slowest decay rate is determined by the largest (in moduli) of the eigenvalues of $T(1)$. In fact, there exist $k>0, \alpha>0$ such that

$$
\|T(t)\|_{\mathcal{L}(X, X)} \leq k e^{-\alpha t}, \quad t \geq 0 .
$$

The iterates of $T(1)$ acting on a bounded set will converge to a compact set. This will not be the case if $r\left(\sigma_{\text {ess }}(T(1))\right)=1$.

Notice that, if (iii) of Theorem 5.2 is satisfied for a given $T$, then it also will be satisfied for any linear dynamical system $\tilde{T}$ on $X$ for which $\|T(1)-\tilde{T}(1)\|$ is sufficiently small. This is not the case in (ii) of Theorem 5.2. A small perturbation of $T$ could make the essential spectral radius $<1$ and change the attractivity properties of the set $\mathcal{A}(T)=\{0\}$.

To apply Theorem 5.2, it is necessary to obtain information about $r\left(\sigma_{\text {ess }}(T(1))\right)$ and the point spectrum $P \sigma(T(1))$ (the eigenvalues of $T(1)$ ). For a discrete dynamical system corresponding to a mapping $\tilde{T}$ on $X$, there is no choice but to determine the spectrum from $\tilde{T}$. If some iterate of $\tilde{T}$ is completely continuous, then $\sigma_{\text {ess }}(\tilde{T})=\{0\}$. This also is the case for a continuous dynamical system for which there is a $T_{1}>0$ such that $T\left(t_{1}\right)$ is completely continuous. 
For linear continuous dynamical systems on $X$, the domain $\mathcal{D}(A)$ of the infinitesimal generator $A: \mathcal{D}(A) \rightarrow X$ is defined to be

$$
\mathcal{D}(A)=\left\{x \in X: \lim _{t \rightarrow 0^{+}} \frac{1}{t}[T(t) x-x] \text { exists }\right\}
$$

and, for $x \in \mathcal{D}(A), A x$ is this limit. In this case, it is known that

$$
P \sigma(T(t))=e^{P \sigma(A) t}
$$

plus possibly zero. Relating $\sigma_{\text {ess }}(T(t))$ to the spectrum of $A$ is much more difficult. In fact, it often is the case that $A$ has only point spectrum and one must have some other way of deducing information about $\sigma(T(t))$. A useful tool is to use known results relating $T(t)$ to the inverse Laplace transform of the resolvent of $A$. Upper bounds on $r\left(\sigma_{e s s}(T(t))\right)$ are easier to obtain and works well from the point of view of asymptotic behavior provided that this bound is $<1$.

The most desirable relation is

$$
\sigma(T(t))=C l_{\mathbb{C}} e^{\sigma(A) t}
$$

plus possibly zero. Conditions for which this is true are now known (see, for example, Henry (1987), van Neerven (1996), Chicone and Latushkin (1999)).

There are interesting examples in applications where both situations (ii) and (iii) in Theorem 5.2 occur. In Section 10, such an example of a linearly damped wave equation with nonnegative damping is presented. In the Appendix, there is an example for a neutral functional differential.

For general dynamical systems $T$ on a general metric space $X$, it perhaps is possible to develop a qualitative theory for the situation in which $\mathcal{A}(T)$ attracts each compact set of $X$. The first step should be the specification of conditions which ensure that such objects exist. The following result is true for general dynamical systems. Everything except part (i) is due to Hale et al. (1972). The statement on connectness is due to Massatt (1983) and on invariantly connected is due to Gobbino and Sardella (1997).

Theorem 5.3. For a dynamical system $T: \mathbf{G}^{+} \times X \rightarrow X$, there is the maximal compact invariant set $\mathcal{A}(T)$ which attracts compact sets of $X$ if and only if there exists a compact set $K$ that attracts compact sets of $X$.

Furthermore, in such a case, $\mathcal{A}(T)=\bigcap_{t \in \mathbf{G}^{+}} T(t) K$, is independent of $K$ and

(i) $\mathcal{A}(T)$ is invariantly connected if $X$ is connected and connected if $X$ is a Banach space. Also, for continuous dynamical systems, $\mathcal{A}(T)$ always is connected.

(ii) $\mathcal{A}(T)$ is stable.

(iii) For any compact set $K$, there is a neighborhood $U_{K}$ of $K$ and a $t_{0}=t_{0}(K)$ such that $\gamma^{+}\left(T\left(t_{0}\right) U_{K}\right)$ is bounded. 
If we assume that the space $X$ is not locally compact, then there is a dynamical system on $X$ which is point dissipative and there is no maximal compact invariant set. For example, the discrete dynamical system defined by a map $T$ which is a retract onto the unit ball satisfies this property. To obtain results based on point dissipativeness, we must restrict the class of dynamical systems.

For a linear dynamical system on a Banach space $X$ which is point dissipative, part (ii) of Theorem 5.2 implies the existence of the maximal compact invariant set (the origin) which attracts compact sets. It perhaps is not to be expected that the same is true for general dynamical systems and, thus, one should impose additional conditions on the dynamical system $T$. We have seen in Theorem 5.1 that, if there is a $t_{1}>0$ such that $T\left(t_{1}\right)$ is completely continuous and point dissipative, then there exists the compact global attractor, which implies much more.

It would be interesting to characterize those dynamical systems with the property that point dissipative implies the existence of a compact set which attracts compact sets.

To obtain other results on the existence of the maximal compact invariant set and the existence of the compact global attractor, we introduce, in a different but equivalent way, the class of asymptotically smooth dynamical systems due to Hale et al. (1972). In the Appendix, we have indicated how the study of neutral functional differential equations served as motivation for this class.

We say that the dynamical system $T(t), t \in \mathbf{G}^{+}$, is asymptotically smooth if, for any bounded set $B$ in $X$ for which $T(t) B \subset B$ for $t \in \mathbf{G}^{+}$, there exists a compact set $J$ in the closure of $B$ such that $J$ attracts $B$.

Notice that, in the definition of asymptotically smooth, there is the conditional hypothesis: "for any bounded set $B$ for which $T(t) B \subset B$ for $t \in \mathbf{G}^{+}$." The definition of asymptotically smooth involves only a qualitative property of the semigroup and imposes nothing on the flow.

The concept of asymptotically smooth is equivalent to asymptotically compact as introduced by Ladyženskaja (1987). It is not the same as the concept of asymptotically compact as used by Ball (1997) or Sell and You (2002) since their definition involves the dynamics of the dynamical system and actually implies that positive orbits of bounded sets are bounded.

Any dynamical system generated by an ordinary differential equation in $\mathbb{R}^{n}$ is asymptotically smooth. Also, if a dynamical system $T$ has the property that there is a $t_{1}>0$ such that $T\left(t_{1}\right)$ is conditionally completely continuous, then it is asymptotically smooth. A mapping $M: C \rightarrow C$ is said to be conditionally completely continuous if, for any bounded $B \subset C$ for which $M B$ is bounded, the closure of $M B$ is compact.

To define another interesting class of asymptotically smooth dynamical systems, we need the Kuratowski measure $\alpha(B)$ of noncompactness of a bounded set $B \subset X$ given by

$$
\alpha(B)=\inf \{d>0: B \text { has a finite cover of diameter }<d\} .
$$

A continuous mapping $S: X \rightarrow X$ is said to be an $\alpha$-contraction on $X$ if there is a 
constant $0 \leq k<1$ such that

$$
\alpha(S B) \leq k \alpha(B) \quad \forall \text { bounded } B \subset X
$$

If $T: \mathbf{G}^{+} \times X \rightarrow X$ is a dynamical system on $X$ and there is a $t_{1}>0$ such that $T\left(t_{1}\right)$ is an $\alpha$-contraction on $X$, then $T$ is asymptotically smooth. A special case which occurs frequently in applications is

$$
T(t)=S(t)+U(t)
$$

where $U(t)$ is conditionally completely continuous for $t \geq 0$ and, if $B \subset X$ is bounded, then dist $(S(t) B, 0) \rightarrow 0$ as $t \rightarrow \infty$. For other classes of asymptotically smooth dynamical systems, see Hale (1987), Raugel (2002).

Theorem 5.4. If the dynamical system $T: \mathbf{G}^{+} \times X \rightarrow X$ is asymptotically smooth, then

(i) $T$ is point dissipative,

(ii) for any compact set $K \subset X$, there is a $t_{0}(K)$ such that $\gamma^{+}\left(T\left(t_{0}\right) K\right)$ is bounded,is equivalent to

(a) there exists the maximal compact invariant set $A$,

(b) A is a local attractor,

(c) for each compact set $K \subset X$, there is a neighborhood $U$ of $K$ such that $A$ attracts $U$.

Condition (i) in Theorem 5.4 does not imply (a)-(c) and, thus, (ii) cannot be eliminated. In fact, Cholewa and Hale (2000) show that there is an asymptotically smooth map on a metric space $X$ which is point dissipative and there are a compact invariant set $K$ and a sequence $n_{j} \rightarrow \infty$ as $j \rightarrow \infty$ such that $\gamma^{+}\left(T^{n_{j}} K\right)$ is unbounded for each $j$.

We say that a dynamical system $T: \mathrm{G}^{+} \times X \rightarrow X$ is compact dissipative if there is a bounded set $B \subset X$ that attracts compact sets of $X$. If $T$ is asymptotically smooth and compact dissipative, then it can be shown (see, for example, Hale (1987)) that there exists a compact invariant set that attracts compact sets of $X$. As a consequence, from Theorem 5.4, we have

Theorem 5.5. If the dynamical system $T: \mathbf{G}^{+} \times X \rightarrow X$ is asymptotically smooth, then compact dissipative implies the statements (a)-(c) in Theorem 5.4.

The example mentioned above of Cholewa and Hale (2000) shows that one cannot replace compact dissipative in Theorem 5.5 by point dissipative. It is interesting to try to determine those dynamical systems for which point dissipative is equivalent to compact dissipative. A reasonable candidate is the class consisting of those systems 
which are $\alpha$-contractions or perhaps those satisfying (2). It is not known at this time whether or not this is true.

On the other hand, Massatt (1981) has shown that point dissipative and compact dissipative are equivalent for those dynamical systems generated by neutral functional differential equations with an exponentially stable $D$ operator (for the definitions, see the Appendix). To obtain such a result, he exploited the fact that the equation defined a dynamical system on two Banach spaces $X_{1}, X_{2}$ with $X_{2}$ compactly imbedded into $X_{1}$ and the representation of each dynamical system had the form (2) with $S(t)$ being a linear semigroup. The details of the technique, which we do not describe, should be applicable to other situations.

Another interesting reason for characterizing those dynamical systems for which point dissipativeness and compact dissipativeness are equivalent is that it would improve significantly the applicability of the following asymptotic fixed point theorem discovered independently and with different proofs by Nussbaum (1972) and Hale and Lopes (1973).

Theorem 5.6. If $X$ is a Banach space and $T: X \rightarrow X$ is compact dissipative, then there is a fixed point of $T$.

There are many important dynamical systems for which the conditions for the existence of a compact global attractor in Theorem 5.1 are not satisfied. The following result characterizes those dynamical systems for which the compact global attractor exists and is applicable to neutral functional differential equations with an exponentially stable $D$ operator as well as a large class of hyperbolic partial differential equations.

Theorem 5.7. A dynamical system $T: \mathbf{G}^{+} \times X \rightarrow X$ has a compact global attractor if and only if

(i) $T$ is asymptotically smooth.

(ii) $T$ is point dissipative.

(iii) For any bounded set $B$ in $X$, there is an $t_{0}=t_{0}(B)$ such that $\gamma^{+}\left(T\left(t_{0}\right) B\right)$ is bounded.

The 'if' part of Theorem 5.7 is essentially due to Hale et al. (1972) taking into account some refinements in the thesis of Cooperman (1978) and the paper of Massatt (1981). The 'only if' part makes use of an example of Cholewa and Hale (2000) in which they show that there is a dynamical system for which there is a compact global attractor and, at the same time, there is a bounded set for which the positive orbit is unbounded (that is, (iii) in the theorem is necessary).

There are other characterizations of those dynamical systems for which there is the compact global attractor (see, for example, Temam (1997)). An absorbing set for a dynamical system $T$ is a bounded set $B_{1}$ in $X$ such that, for any bounded set $U$ in 
$X$, there is an $t_{0}\left(B_{1}, U\right)$ such that $T(t) U \subset B_{1}$ for $t \geq t_{0}\left(B_{1}, U\right)$. The existence of an absorbing set is equivalent the old terminology of uniform ultimate boundedness used in ordinary differential equations (see, for example, Yoshizawa (1966)).

It is easy to prove the following result.

Theorem 5.8. There is a compact global attractor for the dynamical system $T$ : $\mathbf{G}^{+} \times X \rightarrow X$ if and only if

(i) $T$ is asymptotically smooth,

(ii) there exists an absorbing set.

Of course, the hypotheses in Theorems 5.7 and 5.8 must be equivalent. If it is possible to apply Theorem 5.8, then an explicit bound on the compact global attractor is obtained since it must belong to each absorbing set. The verification of the existence of an absorbing set involves the construction of a Lyapunov function which is strictly decreasing for large initial values. Many applications of this method have been given to particular partial differential equations for which there is a natural energy function. The Lyapunov function is then obtained from modifications of the energy function (see, for example, Temam (1997)). In the application of Theorem 5.7, there is no information about an explicit bound on the compact global attractor. However, in many of the applications to partial differential equations, the energy function itself together with Theorem 5.7 can be used to verify the existence of a compact global attractor. This often requires fewer estimates (see, for example, Hale (1983), (1987)).

We remark also that the construction of explicit Lyapunov functions for general systems is difficult if not impossible. This is easily observed in ordinary differential equations. On the other hand, many situations have been discussed where one is able to show point dissipativeness directly. If the dynamical system is point dissipative, then the compact global attractor exists if either $X$ is locally compact or there is $t_{1}$ such that $T\left(t_{1}\right)$ is completely continuous. In more general cases, if it is known that the system is point dissipative, it is still necessary to verify (i) and (iii) in Theorem 5.7 to know that the compact global attractor exists. Thus, an absorbing set exists, but a specific one is not obtained. An explicit example illustrating these remarks is given in Section 7.

\section{Gradient systems}

A typical example of a gradient ordinary differential equation in $\mathbb{R}^{n}$ is

$$
\dot{x}=-\nabla F(x),
$$

where $F: \mathbb{R}^{n} \rightarrow \mathbb{R}$ is at least a $C^{1}$ function. If we define

$$
V: x \in \mathbb{R}^{n} \mapsto F(x)
$$


and $x(t)$ is a solution of (3), then $\dot{V}(x)(t) \equiv \frac{d}{d t} V(x(t))$ satisfies

$$
\dot{V}(x)=-|\nabla F(x)|^{2} \leq 0, \quad x \in \mathbb{R}^{n} .
$$

If $x\left(t, x_{0}\right)$ is the solution of (3) through $x_{0}$ at $t=0$ and $\gamma^{+}\left(x_{0}\right)$ is bounded, then $\omega\left(x_{0}\right)$ is a compact invariant set in $\mathbb{R}^{n}$. A simple argument shows that (this is actually a special case of the LaSalle invariance principle), for any $y \in \omega\left(x_{0}\right)$, the solution $x(t, y)$ belongs to $\omega\left(x_{0}\right)$ and $V(x(t, y))=V(y)$ for all $t \in \mathbb{R}$. As a consequence, $\dot{V}(x(t, y))=0$ for all $t \in \mathbb{R}$ which implies that $\nabla F(x(t, y))=0$ for all $t \in \mathbb{R}$. This is equivalent to saying that $\omega\left(x_{0}\right)$ belongs to the set of equilibria of (3).

The flow for this gradient system will be Morse-Smale if there are only a finite number of equilibria, all of which are hyperbolic and the stable and unstable manifolds intersect transversally.

In this section, we give generalizations to infinite dimensions of gradient systems based upon some unpublished notes of Hale and Raugel (2003) (see, also, Conley (1978), Hurley (1991), (1992), (1995)).

Definition 6.1. Let $T$ be a dynamical system on $\mathbf{Z}^{+} \times X$. For $\epsilon>0$, an $\epsilon$-chain for $T$ is a sequence of points $x_{0}, x_{1}, \ldots, x_{k} \in X$ ( $k$ is supposed to be at least equal to 1 ), such that

$$
d\left(T\left(x_{i}\right), x_{i+1}\right) \leq \epsilon, \quad 0 \leq i \leq k-1 .
$$

This $\epsilon$-chain is said to go from $x_{0}$ to $x_{k}$ and to have length $k$. A point $x \in X$ is called chain recurrent if, for any $\epsilon>0$, there are an integer $k \geq 1$ and an $\epsilon$-chain of length $k$ of points $x_{0}, x_{1}, \ldots, x_{k} \in X$ with $x_{0}=x=x_{k}$.

We denote by $R(T)$ the set of chain recurrent points of $T$. We can define an equivalence relation on $R(T)$ by $x \sim y$ if and only if, for each $\epsilon>0$, there exists an $\epsilon$-chain from $x$ to $y$ and an $\epsilon$-chain from $y$ to $x$.

The equivalence classes for this relation are called the chain-transitive components of $T$. Each chain-transitive component (and, in particular, $R(T)$ ) is easily proved to be closed.

Likewise, let $T: \mathbb{R}^{+} \times X \rightarrow X$ be a dynamical system. For $\epsilon>0$ and $\tau>0$, an $(\epsilon, \tau)$-chain for $T$ of length $k$ ( $k$ is supposed to be at least equal to 1 ), is a sequence of real numbers $t_{0}, t_{1}, \ldots, t_{k-1}, t_{j} \geq \tau$, for any $j$, and of points $x_{0}, x_{1}, \ldots, x_{k} \in X$ such that

$$
d\left(T\left(t_{i}\right) x_{i}, x_{i+1}\right) \leq \epsilon, \quad 0 \leq i \leq k-1 .
$$

As before, a point $x \in X$ is called chain recurrent if, for any $\epsilon>0$ and any $\tau>0$, there are an integer $k \geq 1$ and an $(\epsilon, \tau)$-chain for $T$ of length $k$ of points $x_{0}, x_{1}, \ldots, x_{k} \in X$, $t_{0}, t_{1}, \ldots, t_{k-1} \in \mathbb{R}$ with $x_{0}=x=x_{k}$. As before, we denote by $R(T)$ the set of chain recurrent points of the dynamical system $T$, and define the corresponding equivalence relation on $R(T)$ and the chain-transitive components.

One easily shows that the nonwandering set $\Omega(T)$ satisfies $\Omega(T) \subset R(T)$.

Let $E(T)$ be the set of stationary points of $T$ (also called equilibria or critical points); that is, those $x \in X$ such that $T(t) x=x$ for $t \in \mathbf{G}^{+}$. 
Definition 6.2. A dynamical system $T$ is said to be a gradient-like if

$$
\Omega(T)=E(T)
$$

A dynamical system $T(t), t \in \mathbf{G}^{+}$, is said to be gradient dynamical system if there exists a Lyapunov function $V: X \rightarrow \mathbb{R}$; that is, a function $V: X \rightarrow \mathbb{R}$ satisfying the following properties:

(i) $V(T(t) x) \leq V(x)$ for all $t \in[0, \infty) \cap \mathbf{G}^{+}, x \in X$,

(ii) $V(T(t) x)=V(x)$ for $t \in \mathbf{G}^{+}$implies that $x \in E(T)$.

A dynamical system $T(t), t \in \mathbf{G}^{+}$, is said to be a strongly gradient dynamical system if it is a gradient-like dynamical system and $R(T)=E(T)$.

These concepts are independent.

Example 6.3 (Gradient-like and not gradient). It is possible to show that there is an ordinary differential equation in $\mathbb{R}^{2}$ for which $\omega(x)$ is the origin for each $x \in \mathbb{R}^{2}$ and the compact global attractor $\mathcal{A}$ is homeomorphic to the unit disk and, for each $x \in \mathcal{A}$, $x$ not the origin, the orbit $\gamma(x)$ through $x$ is a homoclinic orbit; that is, $\alpha(x)$ and $\omega(x)$ are the origin. As a consequence, there can be no Lyapunov function since it must be constant on $\gamma(x) \cup \alpha(x) \cup \omega(x)$ (see, for example, Bhatia and Szegö (1967, p. 44), Conley (1978)).

Example 6.4 (Gradient and not strongly gradient). We give two examples. On the circle $S^{1} \cong[0, \pi)$, the dynamical system defined by

$$
\frac{d \theta}{d t}=\cos ^{2} 2 \theta
$$

is gradient-like since $\Omega(T)=E(T)$. It is gradient with Lyapunov function $V(\theta)=$ $-\frac{\theta}{2}+\frac{1}{8} \sin 4 \theta$. However, it is not strongly gradient since $R(T)=S^{1}$.

This fact was pointed out earlier by Conley (1978) using the following example. Consider the system of equations

$$
\frac{d x}{d t}=0, \quad \frac{d y}{d t}=-\left(1-x^{2}\right)\left(1-y^{2}\right)
$$

on the square $\{|x| \leq 1,|y| \leq 1\}$. The boundary of the square is the set of equilibria. The solutions starting at other points run vertically downward to the bottom of the square. Every point $(x, y)$ belongs to $R(T)$. Indeed, for any $\epsilon>0$ and any $(x, y)$ and $\left(x^{*}, y^{*}\right)$ which are not equilibria, there is an $\epsilon$-chain from $(x, y)$ and $\left(x^{*}, y^{*}\right)$; by allowing small errors, we first go down to points close to $\left(x^{*},-1\right)$, then go to points close to $(-1,-1)$. Again, allowing small errors, one then goes up to points close to $(-1,1)$ and to the right to points close to $\left(x^{*}, 1\right)$ and, finally, down to $\left(x^{*}, y^{*}\right)$. 
In Example 6.4, the space $X$ is a compact metric space for which $R(T)=X$. The next example is concerned with a noncompact metric space for which $T$ is gradient-like and $R(T)$ is unbounded.

Example 6.5 (Gradient-like, not gradient, $\Omega(T)$ compact and $R(T)$ unbounded). Cholewa and Hale (2000, Prop. 2.1) have shown that there is a complete metric space $(X, \rho), 0 \in X$, and a map $T: X \rightarrow X$ which is continuous and asymptotically smooth such that $\{0\}$ attracts each point of $X$ and, for any $\epsilon>0$ and any $\ell \geq 1$, the set $\gamma^{+}\left(T^{\ell}(B(0, \epsilon))\right.$ is unbounded, where $B(0, \epsilon)$ is the $\epsilon$-neighborhood of $\{0\}$.

For any $\epsilon>0, R>0$, there is a $z \in B(0, \epsilon)$ such that dist $(T z, 0)>R$. We want to construct an $\epsilon$-chain from $x_{0} \equiv T z$ to $x_{0}$. Choose $x_{1} \in B\left(T x_{0}, \epsilon\right)$. There is an integer $k$ such that $T^{k} x_{1} \in B(\epsilon, 0)$. Define $x_{j+1}=T^{j} x_{1}, 1 \leq j \leq k$ and $x_{k+2}=z$, $x_{k+3}=x_{0}$. This is an $\epsilon$-chain from $x_{0} \equiv T z$ to $x_{0}$. Since $R$ is arbitrary, this shows that $R(T)$ is unbounded.

It always is true that gradient implies gradient-like and in some situations, gradient implies strongly gradient as stated in

Theorem 6.6 (Implication of gradient). If $T(t), t \in \mathbf{G}^{+}$, is a gradient dynamical system on $X$, then it is gradient-like.

If, in addition, $E(T)$ is a finite set, $T(t)$ is a strongly gradient system.

If $T$ admits a compact global attractor, then we have

Theorem 6.7. If $T(t), t \in \mathbf{G}^{+}$, is a strongly gradient dynamical system which admits a compact global attractor $\mathcal{A}$, then it is a gradient system with a Lyapunov function as defined in Definition 6.2.

Theorem 6.8. If $T(t), t \in \mathbf{G}^{+}$, is a gradient-like dynamical system, then

(i) $\gamma^{+}\left(x_{0}\right)$ relatively compact implies that $\omega\left(x_{0}\right) \subset E(T)$.

(ii) If $\gamma^{-}\left(x_{0}\right)$ exists and is relatively compact, then $\alpha\left(\gamma^{-}\left(x_{0}\right)\right) \subset E(T)$.

Corollary 6.9. If $T(t), t \in \mathbf{G}^{+}$, is an asymptotically smooth gradient-like dynamical system, then $\gamma^{+}\left(x_{0}\right)$ bounded implies that $\omega\left(x_{0}\right) \subset E(T)$.

A system may be gradient-like and $\omega\left(x_{0}\right)$ need not be a singleton (Palis and de Melo (1982, p. 15)). One can impose some conditions on the multiplicity of the zero eigenvalue and obtain that $\omega\left(x_{0}\right)$ is a singleton. A special case of a much more general result of Hale and Raugel (1992) is

Theorem 6.10. Suppose that $T: \mathbf{G}^{+} \times X \rightarrow X$ is a gradient-like dynamical system. If $\gamma^{+}\left(x_{0}\right)$ is relatively compact and there is a $y \in \omega\left(x_{0}\right)$ such that either 1 is not in the spectrum $\sigma(D T(1)(y))$ of $D T(1)(y)$ or 1 is in $\sigma(D T(1)(y))$ and $\sigma(D T(1)(y)) \cap S^{1}=$ $\{1\}$ and 1 is a simple eigenvalue, then $\omega\left(x_{0}\right)$ is a singleton. 
Remark 6.11. If $T: \mathrm{G}^{+} \times X \rightarrow X$ is a gradient-like dynamical system on $X$ and we define $\mathcal{A}(T)$ as in (1), then it is easy to verify that

$$
\mathcal{A}(T)=\mathrm{Cl} W^{u}(E(T)) \cap \mathcal{A}(T),
$$

where $W^{u}(E(T))$ is the unstable set of $E(T)$. Furthermore, if each element of $E(T)$ is isolated, then

$$
\mathcal{A}(T)=\operatorname{Cl}\left(\bigcup_{x_{0} \in E(T)} W^{u}\left(x_{0}\right)\right) \cap \mathcal{A}(T) .
$$

If $\mathcal{A}(T)$ is a compact set (that is, the maximal compact invariant set), then we do not need to take the closure in (4) and (5).

If $E(T)$ is a finite set, each $x_{0} \in E(T)$ is hyperbolic and $W^{u}\left(x_{0}\right)$ is a manifold, then $\mathcal{A}(T)$ is the union of a finite number of manifolds.

The exponential attractivity of the attractor is important when proving lower semicontinuity results of maximal invariant sets and compact global attractors as well as obtaining a better understanding of the transient behavior of solutions. Special cases of the result below are implicitly contained in Hale and Raugel (1989), Babin and Vishik (1989). Kostin (1990) proved the same result under the assumption that the compact global attractor exists. The result as stated is an unpublished result of Hale and Raugel.

Theorem 6.12 (Exponential attraction of maximal compact invariant set). Let $T: \mathbf{G}^{+} \times X \rightarrow X$ be a gradient dynamical system on a Banach space $X$ with the property that there is a maximal compact invariant set $\mathcal{A}$ which attracts a neighborhood of each compact set. Also, suppose that the associated Lyapunov function satisfies, for any $t \in \mathbf{G}^{+}, t>0$,

$$
V(T(t) x)<V(x), \quad \forall x \in X \backslash E(T) .
$$

If $T(t), t \in \mathbf{G}^{+}$, is $C^{1}$ on $X$ with each equilibrium hyperbolic, then there exist positive constants $C_{1}, \gamma$ and a neighborhood $B_{0}$ of $\mathcal{A}$ such that

$$
\operatorname{dist}_{X}(T(t) y, \mathcal{A}) \leq C_{1} e^{-\gamma t}, \quad t \in \mathbf{G}^{+}, y \in B_{0} .
$$

Remark 6.13. If the conditions of the preceding theorem are satisfied, then the set $E(T)$ is finite, say $E(T)=\left\{e_{j}, 1 \leq j \leq p\right\}$. One can show that the set $B_{0}$ can be chosen in such a way that, for any $y \in B_{0}$, there is an integer $k$ such that $y \in W^{s}\left(e_{k}\right)$, the stable set of $e_{k}$. Since $T(t) y \rightarrow e_{k}$ as $t \rightarrow \infty$, there are small neighborhoods $U_{j}$ of $e_{j}, 1 \leq j \leq p$, such that there are only a finite number of excursions of $T(t) y$ to the sets $B_{0} \cap U_{j}, j \neq k$, before entering $B_{0} \cap U_{k}$; that is, there is a finite number $q(y)$ of segments $\gamma_{j} \equiv\left\{T(t) y, t \in\left[t_{j}, t_{j+1}\right)\right\}, j=1,2, \ldots, q(y)$, of $\gamma^{+}(y)$ before entering $B_{0} \cap U_{k}$. If we define $\gamma_{q(y)+1}$ as that part of the orbit through $y$ which remains in $B_{0} \cap U_{k}$, then the collection of segments $\left\{\gamma_{j}, j=1,2, \ldots, q(y)+1\right\}$ is referred to as a 
finite-dimensional combined trajectory by Babin and Vishik (1989). Actually, under additional conditions, they show that this finite dimensional trajectory converges exponentially to a finite dimensional trajectory on $\mathcal{A}$ consisting of pieces of invariant manifolds of equilibria; that is, for any $\eta>0$, one can construct a finite-dimensional combined trajectory $u_{\eta}(t) \in \mathcal{A}$ such that $\left\|T(t) y-u_{\eta}(t)\right\|_{X} \leq C(\eta) \exp (-\eta t)$, where $C(\eta)>0$ depends on $\eta$.

\section{A viscoelastic model}

As remarked earlier, dynamical systems which model physical processes depend upon many parameters. To simplify the model, one chooses the smallest number of parameters which reflects well the experimental observations of the process. It thus becomes important to analyze the dynamics as a function of the parameters; that is find those values of the parameters for which the system is $\mathcal{A}$-stable as well as those for which there are bifurcations.

In this section, we present a model in viscolasticity which will illustrate the role of parameters in the discussion of the properties of the flow on the compact global attractor.

Suppose that $\delta>0$ is a constant, $a$ and $g$ are functions which satisfy the following conditions:

$$
\begin{aligned}
& a \in C^{2}([0, \delta], \mathbb{R}), a(0)=1, a(\delta)=0, a(s)>0, \text { for } s \in[0, \delta), \\
& g \in C^{1}(\mathbb{R}, \mathbb{R}),
\end{aligned}
$$

and consider the scalar functional differential equation

$$
\dot{x}(t)=-\int_{-\delta}^{0} a(-\theta) g(x(t+\theta)) d \theta .
$$

Let $C=C([-\delta, 0], \mathbb{R})$. For any $\varphi \in C$, it can be shown that there is a unique continuous function $x(\cdot, \varphi)$ defined on a maximal interval $\left[-\delta, \alpha_{\varphi}\right)$ with the property that $x(\theta, \varphi)=\varphi(\theta)$ for $\theta \in[-\delta, 0]$ and $x(t, \varphi)$ satisfies $(7)$ for $t \in\left[0, \alpha_{\varphi}\right)$. If we define

$$
\left(T_{a, g}(t) \varphi\right)(\theta)=x(t+\theta, \varphi), \quad \theta \in[-\delta, 0], \quad t \in\left[0, \alpha_{\varphi}\right),
$$

then $T_{a, g}(t): C \rightarrow C$ is a local dynamical system. If each solution is defined for all $t \in[-\delta, \infty)$, then $T_{a, g}: \mathbb{R}^{+} \times C \rightarrow C$ is a dynamical system on $C$.

If $c$ is a real constant, we abuse notation by letting $c$ denote the constant function in $C$ with value $c$ at every point in $[-\delta, 0]$. The equilibria of $T_{a, g}$ are the zeros of $g$.

Levin and Nohel (1964) introduced (6) as a model in viscoelasticity under the assumption that $a$ satisfies

$$
a^{\prime}(s) \leq 0, \quad a^{\prime \prime}(s) \geq 0, \quad s \in[0, \delta]
$$


and $g$ satisfies

$$
G(x)=\int_{0}^{x} g(u) d u \rightarrow \infty \text { as }|x| \rightarrow \infty .
$$

They proved that the limit of a solution must be an equilibrium if $a$ is a nonlinear function and possibly a periodic solution of period $\delta$ if $a$ is linear. Using the concepts of limit sets and invariance in dynamical systems, it is possible to prove much more (see Hale (1965), Hale et al. (2002)).

Theorem 7.1 (Nonlinear kernel). If a is a nonlinear function satisfying (6), (8) and $g$ satisfies (9), then

(i) $T_{a, g}$ is gradient,

(ii) if the zeros of $g$ are bounded (that is, the set $E_{g}$ of equilibria is bounded), then the compact global attractor $\mathcal{A}_{a, g}$ exists,

(iii) for any $\varphi \in C$, there is a zero $c_{\varphi}$ of $g$ such that $T(t) \varphi \rightarrow c_{\varphi}$ as $t \rightarrow \infty$.

If the zeros of $g$ are simple, then

(iv) each equilibrium is hyperbolic. If $g(c)=0$, then $c$ is hyperbolically stable (that is, $c$ is hyperbolic and attracts exponentially a neighborhood of itself) if and only if $g^{\prime}(c)>0$ and hyperbolically unstable (that is, hyperbolic and unstable) with $\operatorname{dim} W^{u}(c)=1$ if and only if $g^{\prime}(c)<0$ and the attractor $\mathcal{A}_{a, g}$ is the union of a finite number of one dimensional manifolds,

$$
\mathcal{A}_{a, g}=\bigcup_{c \in E_{g}} W^{u}(c)
$$

Indication of the proof. The proof is based on the construction of a Lyapunov function and then using the fact that this function must be constant on compact invariant sets.

Since $a$ is $C^{1}$, the solution of (7) through $\varphi$ must have $\ddot{x}(t)$ continuous for $t \in$ $\left[0, \alpha_{\varphi}\right)$. A few elementary calculations show that $x(t)$ must satisfy the equation

$$
\ddot{x}(t)+g(x(t))=-a^{\prime}(\delta) H_{\delta, g}\left(x_{t}\right)+\int_{-\delta}^{0} a^{\prime \prime}(-\theta) H_{\theta, g}\left(x_{t}\right) d \theta,
$$

where we are using the notation

$$
H_{s, g}(\psi)=\int_{-s}^{0} g(\psi(\theta)) d \theta, \quad s \in[0, \delta] .
$$

If we define $V: C \rightarrow \mathbb{R}$ as

$$
V(\varphi)=G(\varphi(0))-\frac{1}{2} \int_{-\delta}^{0} a^{\prime}(-\theta)\left[H_{-\theta, g}(\varphi)\right]^{2} d \theta
$$


where $G(x)=\int_{0}^{x} g(u) d u$, then the derivative of $V$ along solutions of (7) is given by

$$
\dot{V}(\varphi)=\frac{1}{2} a^{\prime}(\delta)\left[H_{\delta, g}(\varphi)\right]^{2}-\frac{1}{2} \int_{-\delta}^{0} a^{\prime \prime}(-\theta)\left[H_{-\theta, g}(\varphi)\right]^{2} d \theta \leq 0
$$

Condition (9), relation (12) and the definition of $G$ imply that each solution is defined for all $t \geq-\delta$ and, thus, $T_{a, g}$ is a dynamical system on $C$. Also, positive orbits of bounded sets are bounded.

For any solution $x(t)$ of $(7)$, we have observed that $\ddot{x}(t)$ is continuous for $t \geq 0$. The Arzela-Ascoli Theorem implies that $T_{a, g}(t)$ is completely continuous for $t \geq \delta$.

Let $\Omega(a, g)$ be the nonwandering set of $(7)$. If $\varphi \in \Omega(a, g)$, then the solution $x(t, \varphi)$ through $\varphi$ at $t=0$ must have the property that $x_{t}(\cdot, \varphi) \in \Omega(a, g)$ for $t \in[0, \infty)$. Since $x(t, \varphi)$ satisfies (10) and $\dot{V}$ satisfies (12) and is $\leq 0$, it is easy to deduce that

$$
\Omega(a, g) \subset S=\left\{\psi \in C: H_{\delta, g}(\psi)=0 \text { if } a^{\prime}(\delta) \neq 0, H_{s, g}(\psi)=0 \text { if } a^{\prime \prime}(s) \neq 0\right\},
$$

Furthermore, a solution $x(t, \varphi)$ through $\varphi \in \Omega(a, g)$ must satisfy the ODE

$$
\ddot{x}(t)=g(x(t)) .
$$

Since $a$ is not a linear function, there are an $s_{0}, a^{\prime \prime}\left(s_{0}\right) \neq 0$ and an interval $I_{s_{0}}$ containing $s_{0}$ such that $a^{\prime \prime}(s) \neq 0$ for $s \in I_{s_{0}}$. If $x(t)$ is a solution of $(7)$ for which $x_{t} \in \Omega(a, g)$, then $x(t)$ satisfies (13). Since $H_{s, g}\left(x_{t}\right)=0$ for $t \in(0, \infty), s \in I_{s_{0}}$, it follows that $\dot{x}(t)=\dot{x}(t-s)$ for $s \in I_{s_{0}}$. As a consequence, $\dot{x}(t)$ is a constant. Since $x_{t} \in \Omega(a, g)$, it follows that $x(t)$ is an equilibrium of $(7)$. This proves that (7) is gradient.

If the conditions in (ii) are satisfied, then $T_{a, g}$ is point dissipative. We may now apply Theorem 5.6 to obtain the existence of the compact global attractor.

A careful but not difficult analysis of the linear variational equation about an equilibrium $c$ shows that $c$ is hyperbolic if and only if $g^{\prime}(c) \neq 0$ and the conclusions in (iv) hold.

If an equilibrium $c$ is not hyperbolic, then the eigenvalue zero is simple and the conclusion in (iii) follows from Theorem 10.5.

Supposing that the compact global attractor $\mathcal{A}_{a, g}$ of (4.2) exists and the zeros of $g$ are simple, we describe more detailed properties of the flow. There must be an odd number of elements in $E_{g}$ which can be ordered as $c_{1}<c_{2}<\cdots<c_{2 k+1}$ with each $c_{2 j+1}$ hyperbolically stable and each $c_{2 j}$ hyperbolically unstable with $\operatorname{dim} W^{u}\left(c_{2 j}\right)=1$ for each $j$. To understand the flow on $\mathcal{A}_{a, g}$, it is only necessary to know the limit sets of the unstable manifolds of the saddle points $c_{2 j}, j=1,2, \ldots, k$.

If there is only one equilibrium, then it is the compact global attractor.

If there are only three equilibria, $c_{1}<c_{2}<c_{3}$, then $c_{1}, c_{3}$ are hyperbolically stable and $c_{2}$ is a saddle with a one dimensional unstable manifold. Since the set $\mathcal{A}_{a, q}$ is connected, the set $\mathcal{A}_{a, g}$ is a one-dimensional manifold with boundary given by the 
closure of $W^{u}\left(c_{2}\right)$; that is, there are orbits $\gamma_{1}, \gamma_{3} \subset W^{u}\left(c_{2}\right)$ such that $\alpha\left(\gamma_{1}\right)=c_{2}=$ $\alpha\left(\gamma_{3}\right), \omega\left(\gamma_{1}\right)=c_{1}, \omega\left(\gamma_{3}\right)=c_{3}$. The point $c_{2}$ 'lies between' $c_{1}$ and $c_{2}$ on the attractor $\mathcal{A}_{a, g}$. The ordering of the equilibria on the real line is preserved by the ordering induced by the flow on the attractor.

If there are exactly five equilibria $c_{1}<c_{2}<c_{3}<c_{4}<c_{5}$, then $c_{2}, c_{4}$ are hyperbolically unstable (saddle points) with one dimensional unstable manifolds and the other points are stable. From the connectedness of $\mathcal{A}_{a, q}$, the set $\mathcal{A}_{a, q}$ is the closure of the union of the unstable manifolds of $c_{2}$ and $c_{4}$. If the flow on the attractor preserves the ordering on the real line of the stationary points, then $\omega\left(W^{u}\left(c_{2}\right) \backslash\left\{c_{2}\right\}\right)=\left\{c_{1}, c_{3}\right\}$ and $\omega\left(W^{u}\left(c_{4}\right) \backslash\left\{c_{4}\right\}\right)=\left\{c_{3}, c_{5}\right\}$. Intuitively, this should be the case, but it is not. To state a precise result, it is convenient to introduce the symbol $j[k, \ell]$, where $j$ is one of the saddle points $c_{2}, c_{4}$, to designate that there exist orbits $\gamma_{j k}, \gamma_{j \ell}$ in $W^{u}\left(c_{j}\right)$ such that $\omega\left(\gamma_{j k}\right)=c_{k}, \omega\left(\gamma_{j \ell}\right)=c_{\ell}$; that is, there is an orbit of (7) which connects the equilibrium point $c_{j}$ to $c_{k}$ and an orbit which connects $c_{j}$ to $c_{\ell}$.

The following result is due to Hale and Rybakowski (1982).

Theorem 7.2. Suppose that the conditions of Theorem 7.1 are satisfied. Let $c_{1}<$ $c_{2}<c_{3}<c_{4}<c_{5}$ be given real numbers and consider the class $\mathcal{G}$ of functions $g$ satisfying (6) with the zeros of $g$ being the set $\left\{c_{j}, 1 \leq j \leq 5\right\}$ with each zero being simple (and therefore corresponding to a hyperbolic equilibrium point of (7)). In the class $\mathcal{G}$, each of the following situations for the flow on $\mathcal{A}_{a, g}$ can be realized:

(i) $2[1,3], \quad 4[3,5]$,

(ii) $2[1,4], \quad 4[3,5]$,

(iii) $2[1,5], \quad 4[3,5]$,

(iv) $2[1,3], 4[2,5]$,

(v) $2[1,3], \quad 4[1,5]$.

In either of the cases (i), (iii) or (v), the system is Morse-Smale. Suppose that $\left(a_{0}, g_{0}\right)$ are given functions such that either (i), (iii) or (v) occurs. Since $\left(a_{0}, g_{0}\right)$ is Morse-Smale, it is $\mathcal{A}$-stable with respect to the class of functions $(a, g)$ for which $g$ is close to $g_{0}$ and has exactly 5 equilibria and $a$ is close to $a_{0}$ in the $C^{2}$ topology. It is not necessary to require that $a$ satisfy the monotonicity condition (8). This is important because it is not feasible that one can assert that the kernel $a_{0}$ is known with such accuracy.

The only flow which preserves the natural order on the reals of the equilibria is case (i). Cases (ii) and (iv) are not Morse-Smale since case (ii) has a nontransverse intersection of the unstable manifold $W^{u}\left(c_{2}\right)$ of the saddle point $c_{2}$ and the stable manifold $W^{s}\left(c_{4}\right)$ of the saddle point $c_{4}$ and case (iv) has a nontransverse intersection of $W^{s}\left(c_{2}\right)$ and $W^{u}\left(c_{4}\right)$.

The proof of this theorem in Hale and Rybakowski (1982) shows the existence of the nontransversal intersection of two saddle points with the functions $g$ being 
analytic. However, the proof does not show that the transversality of the stable and unstable manifolds is generic in the class $\mathcal{G}$; that is, in the cases (ii) and (iv), it has not been shown that a small perturbation of $g$ will break the connection between the saddles. It may be that one must perturb the function $a$ out of the class of monotone funtions (8) in order to obtain transversality, but this has not been shown.

Theorem 7.3 (Linear kernel). If a is the linear function satisfying $a(0)=1$, $a(\delta)=0, g$ satisfies (9) and the zeros of $g$ are bounded (that is, the set $E_{g}$ of equilibria is bounded) then

(i) the compact global attractor $\mathcal{A}_{a, g}$ exists.

(ii) If there are no $\delta$-periodic orbits of the ODE (13) and the zeros of $g$ are simple, the same conclusions as in Theorem 7.1 hold for the attractor $\mathcal{A}_{a, g}$.

(iii) If there is a $\delta$-periodic orbit of (13) then it belongs to $\mathcal{A}_{a, g}$, but it can never be hyperbolic in the class of a satisfying (6), (8).

The proof of this result is similar to the proof of Theorem 7.1 with details in Hale et al. (2002).

We remark that explicit estimates of the size of the global attractor (and thus estimates of absorbing sets) are not obtainable from the Lyapunov function given in (11). It is not known if there is a Lyapunov function which will yield an explicit absorbing set.

There are certainly functions $g$ and linear functions $a$ satisfying conditions for which there are $\delta$-periodic orbits of (13). If this is the case, none of the periodic orbits can be hyperbolic. In fact, if it were, then it would be a periodic orbit of $(7)$ for a small perturbation of $a$ in the class (6), (8). On the other hand, if $a$ is nonlinear and satisfies $(6),(8)$, then there are no periodic orbits.

This implies that, in the class of functions $(a, g)$ satisfying $(6),(8),(9)$, no dynamical system generated by system (7) can be Morse-Smale if the dynamical system has a periodic orbit. In particular, there is no generic Hopf bifurcation in this class. The chosen parameter space for $(a, g)$ is too small to capture all of the important dynamics that could occur in a more realistic model. If the class of $a$ is enlarged so that $a^{\prime \prime}(s)$ changes sign, then it is shown in Hale et al. (2002) that a generic Hopf bifurcation can occur. This involves either using normal form theory to find the approximate flow on a center manifold or determining an approximation to the bifurcation function for periodic orbits.

\section{A scalar 1-D parabolic equation}

In this section, we discuss a scalar parabolic equation on a bounded interval with separated boundary conditions. A more general survey of some of the literature on this topic is contained in the Butler lectures of Hale (1997). 
Consider the equation

$$
u_{t}=u_{x x}+f\left(x, u, u_{x}\right) \quad \text { in } \Omega=(0,1),
$$

with the boundary conditions

$$
\left.u_{x}\right|_{x=0}=0=\left.u_{x}\right|_{x=1},
$$

where the function $f$ is assumed to be $C^{2}$.

The results mentioned below are valid for a more general situation; for example there could be a positive space dependent diffusion coefficient and more general boundary conditions. Only the simplest case is presented.

We assume that there is an $s$ such that (14), (15) defines a semigroup $T(t)$ on $H^{s}(0,1)$ and that, if $B$ is a bounded set in $H^{s}(0,1)$ and if there is a $t>0$ such that $T(t) B$ is bounded, then the closure of $T(t) B$ is compact. If $f$ is independent of $u_{x}$ and is a $C^{2}$-function, we can take $s=1$. If $f$ depends upon $u_{x}$, then s depends on the growth rate of $u_{x}$ for large $u_{x}$. If the growth rate is less than cubic, then we can take $s=2$.

We also suppose that the solutions of the initial value problem for the ordinary differential equation

$$
y^{\prime \prime}=f\left(x, y, y^{\prime}\right), \quad y\left(x_{0}\right)=y_{0}, y^{\prime}\left(x_{0}\right)=y_{0}^{\prime},
$$

are defined for $0 \leq x \leq 1,0 \leq x_{0} \leq 1, y_{0} \in \mathbb{R}, y_{0}^{\prime} \in \mathbb{R}$.

Theorem 8.1 (Zelenjak (1968)). System (14), (15) is gradient. Furthermore, if $\gamma^{+}(\varphi)$ is a bounded orbit, then $\omega(\varphi)$ is a singleton.

The nontrivial proof depends upon showing that the hypotheses imply the existence of a Lyapunov function as in Definition 6.2. The simplest way to prove the last part of the theorem (which is different from Zelenjak) is to note the implications of Sturm-Liouville theory of second order equations and use Theorem 10.5.

The dynamical system generated by $(14),(15)$ is completely continuous for $t>0$. As a consequence of the fact that the solution operator of $(14),(15)$ is compact and that the system is gradient, Theorem 5.1 implies the following result.

Theorem 8.2. If system (14), (15) is point dissipative, then there exists the compact global attractor $\mathcal{A}_{f}$. If, in addition, the equilibria are hyperbolic, then

$$
\mathcal{A}_{f}=\bigcup_{\varphi \in E} W^{u}(\varphi) .
$$

It is rather remarkable that transversality between stable and unstable manifolds of hyperbolic equilibria holds for every system (14), (15) (Henry (1985), Angenent (1986)) as stated in 
Theorem 8.3. For any $C^{2}$-function $f$, if $\varphi, \psi$ are hyperbolic equilibria of (14), (15), then $W^{u}(\varphi)$ is transversal to $W^{s}(\psi)$.

If, in addition, (14), (15) is point dissipative, then system (14), (15) is MorseSmale if and only if the equilibria are hyperbolic.

The proof of Theorem 8.3 involves several basic properties of the solutions of (14), (15). Any orbit $\gamma(x)$ having $\alpha(x)=\varphi$ and $\omega(x)=\psi$ must have the property stated in

Lemma 8.4. If $\varphi, \psi$ are hyperbolic equilibria of (14), (15), and there is an orbit $\gamma$ through a point $\eta_{0}$ with $\alpha\left(\eta_{0}\right)=\varphi$ and $\omega\left(\eta_{0}\right)=\psi$, then $\operatorname{dim} W^{u}(\varphi)>\operatorname{dim} W^{u}(\psi)$.

The idea for the proof of Lemma 8.4 is the following. For any function $v(t, x)$, $t \in \mathbb{R}, x \in(0,1)$, let $z(v(t, \cdot))$ be the number of zeros of $v(t, x)$ in $(0,1)$. The function $z(v(t, \cdot)$ is called the zero number or lap number of $v(t, \cdot)$. If $\eta(t)$ is a solution of (14), (15) with $\alpha(\eta)=\varphi, \omega(\eta)=\psi$, then the linear variational equation around $\eta$ is a linear equation of the form

$$
v_{t}=v_{x x}+b(t, x) v+c(t, x) v_{x}
$$

with the coefficients converging exponentially as $t \rightarrow+\infty$ (resp. $t \rightarrow-\infty$ ) to the linear variational equation about $\psi($ resp. $\varphi$ ). As a consequence of this fact and the fact that no solution of (16) can approach zero faster than any exponential, it is to be expected that the zero number $z(\eta(t))$ of $\eta(t)$ should be at least the number of zeros of the eigenfunction corresponding to the largest eigenvalue of the linear variational equation about $\psi$ which, by the Sturm-Liouville theory, is at least $\operatorname{dim} W^{u}(\psi)$. By the same reasoning, for large negative $t, z(\eta(t))$ should be no more that $\operatorname{dim} W^{u}(\varphi)-1$.

The proof of the lemma is completed by a famous result of Nickel (1962), Matano (1982), that asserts that the zero number of solutions of (16) is a nonincreasing function of $t$.

To complete the proof of Theorem 8.3, one uses the characterization of the tangent space $T W^{s}(\psi)$ of $W^{s}(\psi)$ as those functions which are orthogonal to the solutions of the adjoint linear variational equation

$$
w_{t}=-w_{x x}-b(-t, x) w-c(-t, x) w_{x}
$$

which approach $T W^{u}(\varphi)$ as $t \rightarrow \infty$. If $W^{u}(\varphi)$ is not transversal to $W^{s}(\psi)$, then there is a solution of (17) which approaches zero as $t \rightarrow \pm \infty$. One now proves that Lemma 8.4 is valid for $(17)$ and deduces that $\operatorname{dim} W^{u}(\varphi)<\operatorname{dim} W^{s}(\psi)$ which is a contradiction.

As noted in Theorem 8.3, (14) generates a Morse-Smale dynamical system if the compact global attractor exists and the equilibria are hyperbolic. It has been shown independently by Smoller and Wasserman (1984) and by Brunovský and Chow (1984) that the equilibria are hyperbolic in an open dense set of $C^{1}$-functions $f(u)$. 
In the remainder of this section, we assume that each element of the set $E$ of equilibria is hyperbolic and that the compact global attractor $\mathcal{A}_{f}$ exists.

To understand more about the flow on the attractor, we must have some criterion for deciding how the equilibria are connected by orbits. Given $\varphi, \psi \in E$, let $C(\varphi, \psi)$ denote the set of connecting orbits from $\varphi$ to $\psi$; that is, the set of trajectories $u(t)$ which are defined for $t \in \mathbb{R}$ with the property that $\omega(u(\cdot))=\psi$ and $\alpha(u(\cdot))=\varphi$. It is clear that

$$
\mathcal{A}_{f}=E \cup\left(\bigcup_{\varphi, \psi \in E} C(\varphi, \psi)\right) .
$$

It is rather remarkable that the decision about connecting orbits can be reduced to an algebraic problem involving a permutation. If $E=\left\{\varphi_{1}, \ldots, \varphi_{k}\right\}$, then there is a natural order of these elements so that

$$
\varphi_{1}(0)<\varphi_{2}(0)<\cdots<\varphi_{k}(0) .
$$

By uniqueness of the initial value problem for the ODE obtained by setting $u_{t}=0$ in (14), these values are distinct. At the other boundary point $x=1$, the natural order may have changed and we have a permutation $\pi_{f}$ of the integers $1,2, \ldots, k$ defined by

$$
\varphi_{\pi_{f}(1)}(1)<\varphi_{\pi_{f}(2)}(1)<\cdots<\varphi_{\pi_{f}(k)}(1) .
$$

This permutation was introduced by Fusco and Rocha (1991) and some important consequences were deduced. The complete theory was developed by Fiedler and Rocha (1996), (1999), (2000).

Theorem 8.5 (Fiedler and Rocha (1996)). If $\pi_{f}$ is the permutation defined by (18), (19), then $\pi_{f}$ determines, in an explicit constructive process, which equilibria are connected and which are not; that is, this permutation determines which of the sets $C(\varphi, \psi)$ are nonempty.

The proof is constructive and easy to apply to examples (see Fiedler and Rocha (1996) or Hale (1997)).

The orbit structure of (14) is completely determined by the permutation as stated in

Theorem 8.6 (Fiedler and Rocha (2000)). Suppose that $\mathcal{A}_{f}$ (resp. $\mathcal{A}_{g}$ ) is the compact global attractor for (14) associated with reaction function $f$ (resp. g) and let $\pi_{f}$ (resp. $\pi_{g}$ ) be the corresponding permutations. Then the dynamical system defined by $f$ is equivalent to the dynamical system defined by $g$ if and only if $\pi_{f}=\pi_{g}$.

The term meander was introduced by Arnol'd (1988) to denote a connected oriented non-self-intersecting curve in the plane intersecting a fixed oriented base line in $k$ points. The intersections are assumed to be strict crossings. The permutation defined by ordering the intersection points, first along the base points and then along 
the meander, is called a meander permutation. For a fixed $k$, let $S(k)$ be the set of meander perturbations.

For any $\pi \in S(k)$, one can define an index vector $\left(i_{1},, i_{2}, \ldots, i_{k}\right)$ by the relations

$$
\begin{aligned}
i_{1} & =0 \\
i_{n+1} & =i_{n}+(-1)^{n+1} \operatorname{sign}\left[\pi^{-1}(n+1)-\pi^{-1}(n)\right]
\end{aligned}
$$

for $1 \leq n \leq k$. An element $\pi \in S(k)$ is said to be Morse if its index vector satisfies $i_{n} \geq 0$ for $1 \leq n \leq k$. The motivation for the above definition of the index vector comes from expression for the Morse indices of equilibria contained in Fiedler and Rocha (1996).

The permutation $\pi_{f}$ associated with the equilibria of (14) on the $\mathcal{A}_{f}$ is a meander permutation. From the point of view of modeling, it is interesting to determine which meandering permutations can be realized by the permutations obtained from (14).

A permutation $\pi \in S(k)$ with $k$ odd is called dissipative if $\pi(1)=1$ and $\pi(k)=k$. We say that a permutation $\pi \in S(k)$ is realizable by (14) if there is an $f$ such that the compact global attractor $\mathcal{A}_{f}$ exists, each equilibrium is hyperbolic and

$$
\pi=\pi_{f}
$$

Theorem 8.7 (Fiedler and Rocha (1999)). A permutation $\pi \in S(k)$ is realizable by (14) if and only if $k$ is odd and $\pi$ is a dissipative Morse meander.

These results give a complete understanding of the qualitative behavior of the flow for a dissipative parabolic equation in one space dimension with separated boundary conditions. As remarked earlier, there is a constructive procedure for determining the flow on the compact global attractor. Many applications of the results have been given.

\section{A scalar parabolic equation in $N-\mathrm{D}$}

Suppose that $\Omega$ is a bounded domain in $\mathbb{R}^{N}$ with smooth boundary, $f: \bar{\Omega} \times \mathbb{R} \rightarrow \mathbb{R}$ is a $C^{1}$-function and consider the scalar reaction diffusion equation

$$
\begin{aligned}
\partial_{t} u & =\Delta u+f(x, u), \quad t>0, \quad x \in \Omega, \\
u & =0, \quad t>0, \quad x \in \partial \Omega .
\end{aligned}
$$

There is a $p>N$ such that (20) defines a local dynamical system $T$ on the Sobolev space $X=W_{0}^{1, p}(\Omega)$. The dynamical system satisfies the following property: for a bounded set $B$, if there is a $t>0$ such that $T(t) B$ is bounded, then the closure of $T(t) B$ is compact. In particular, the $\omega$-limit set of any bounded positive orbit of (20) is a compact, invariant, connected set in $X$. 
The energy functional

$$
V(\varphi)=\int_{\Omega}\left(\frac{1}{2}|\nabla \varphi(x)|^{2}-F(x, \varphi(x))\right) d x,
$$

where $F(x, u)=\int_{0}^{u} f(x, s) d s$, is such that, if $u(t, x)$ is a solution of $(20)$, then

$$
\dot{V}\left(u(t, \cdot)=-\int_{\Omega}\left|\partial_{t} u(t, x)\right|^{2} d x \leq 0 .\right.
$$

As remarked above, if $\gamma^{+}(\varphi)$ is a bounded orbit, then $\omega(\varphi)$ is a nonempty compact invariant connected set. Relation (21) implies that $\omega(\varphi) \subset E_{f}$, where $E_{f}$ is the set of equilibria; that is, the solutions of

$$
\begin{aligned}
& 0=\Delta \varphi+f(x, \varphi), \quad x \in \Omega, \\
& u=0, \quad x \in \partial \Omega .
\end{aligned}
$$

Theorem 9.1. System (20) is gradient. If $E_{f}$ is bounded and each solution is defined and bounded for $t \geq 0$, then the compact global attractor $\mathcal{A}_{f}$ exists. A sign condition on $f(x, u)$ for large $u$ will ensure that positive orbits of points are bounded.

In the previous section, we have seen that the dynamical system defined by a scalar parabolic equation in one space dimension with separated boundary conditions satisfies the following properties:

(i) it is gradient,

(ii) each bounded orbit converges to a single equilibrium,

(iii) stable and unstable manifolds of hyperbolic equilibria always intersect transversally.

(iv) assuming the existence of a compact global attractor, the system is Morse-Smale if and only if the equilibria are hyperbolic.

These properties hold for each $C^{1}$-function $f\left(x, u, u_{x}\right)$ as well as for much more general boundary conditions.

It is interesting to investigate the extent to which these properties hold when the spatial domain has dimension $>1$.

Regarding (ii), we have the following important result of Simon (1983).

Theorem 9.2. If $f$ in (20) is analytic, then the $\omega$-limit set of any bounded orbit is a singleton.

If $f$ is not analytic, this may not be the case as the following result of Poláčik and Rybakowski (1996) shows. 
Theorem 9.3. For $\Omega$ the unit ball in $\mathbb{R}^{2}$, there is a $C^{1}$-function $f(x, u)$ such that the system (20) has a bounded orbit for which the $\omega$-limit set is a circle of equilibria.

Suppose that the compact global attractor $\mathcal{A}_{f}$ exists and let $U$ be a bounded neighborhood of $\mathcal{A}_{f}$. Brunovský and Poláčik (1997) have shown that system (20) is generically Morse-Smale as stated in

Theorem 9.4. There is a residual set $\mathcal{R} \subset C^{1}(\bar{\Omega} \times \bar{U}, \mathbb{R})$ such that, for every $f \in \mathcal{R}$, system (20) is Morse-Smale; that is, each element of $E_{g}$ is hyperbolic and the stable and unstable manifolds intersect transversally

Theorem 9.4 asserts that Morse-Smale systems are generic in the parametrized family of dynamical systems with the parameter space being $\Lambda=C^{1}(\bar{\Omega} \times \bar{U}, \mathbb{R})$.

It is natural to ask if the parameter set can be restricted to the class $C^{1}(\bar{U}, \mathbb{R})$; that is, to functions $f$ in (20) which do not depend upon the spatial variable. The following result of Poláčik (1999) shows that this is not the case.

Theorem 9.5. There is a smooth bounded convex domain $\Omega \subset \mathbb{R}^{2}$ and a quadratic function $f_{0}(u)=\mu u-u^{2}$ such that for any $C^{1}$-function $f$ with

$$
\left\|f-f_{0}\right\|_{C^{1}([-1,1])} \equiv \sup _{u \in[-1,1]}\left(\left|f_{0}(u)-f(u)\right|+\left|f_{0}^{\prime}(u)-f^{\prime}(u)\right|\right)
$$

sufficiently small, (20) has exactly two hyperbolic equilibria $\varphi$ and $\psi$ such that $W^{u}(\varphi)$ and $W^{s}(\psi)$ intersect nontransversally.

For $(20)$ with $f(x, u)$, we have observed that the system is gradient. However, if we allow the functions $f$ to depend upon the gradient of $u$, then the system may not be gradient. The following result shows much more and is the culmination of much effort by Poláčik (1991), (1995), Poláčik and Rybakowski (1995), (1996) and Prizzi (1998).

Theorem 9.6. Consider the system

$$
\begin{aligned}
\partial_{t} u & =\Delta u+f(x, u, \nabla u), \quad t>0, \quad x \in \Omega, \\
u & =0, \quad t>0, \quad x \in \partial \Omega .
\end{aligned}
$$

in the space $W^{1, p}$ for an appropriate $p>N$, where $f(x, u, w)$ is a $C^{1}$-function.

For any given $p$-dimensional ODE

$$
\dot{y}=g(y),
$$

with $g \in C^{1}\left(\mathbb{R}^{p}, \mathbb{R}^{p}\right)$ there is an $N-D$ parabolic equation (22) with a center manifold on which the flow contains the flow of the ODE (23).

Generically, one can be assured that bounded positive orbits converge to a singleton as stated in (Hirsch (1988), Smith and Thieme (1991)) 
Theorem 9.7. If $f(x, u, \nabla u)$ is a $C^{1}$-function, then there is an open dense set $\mathcal{H}$ of initial data such that, for any $u_{0} \in \mathcal{H}$ with $\gamma^{+}\left(u_{0}\right)$ bounded, $\omega\left(u_{0}\right)$ is a singleton.

Note that this result holds for very general parabolic equations which are not gradient. This implies that the complicated orbits referred to in Theorem 9.3 must be unstable. Knowing that such orbits exist is still very important for the global dynamics. For example, if there are only two stable equilibria, then these orbits are on the boundary of the basins of attraction of each. As a consequence, if the initial data is close to this boundary, then it has an important effect on the transient behavior of the orbit.

\section{Linearly damped wave equation}

The dynamical systems discussed in Sections 8 and 9 have the property that there is a $t_{1}>0$ such that $T\left(t_{1}\right)$ is completely continuous. As a consequence, we could obtain the existence of the compact global attractor by assuming only point dissipativeness. There are many situations where this type of smoothing does not occur and we need to use Theorem 5.7. In this section, we discuss one such dynamical system; namely the linearly damped nonlinear wave equation on a bounded domain. Another important parameter that enters into the problem is the nature of the linear damping. The simplest case is constant damping, but it also is very important to understand local damping when the support of the damping is not all of the domain.

We begin by discussing a linear equation to understand conditions which will ensure the exponential stability of the origin. We then present a rather complete theory for the nonlinear equation with constant damping and, finally, a few results on the case of local damping.

Much of the presentation is based on Hale and Raugel (1993) which was heavily influenced by Hale (1983), (1987). Our objective is to stress some of the main ideas and often present results under hypotheses which are more restrictive than necessary.

\subsection{Linearly damped linear wave equation}

Let $\Omega$ be a bounded domain in $\mathbb{R}^{n}, n \leq 3$ with Lipschitz boundary, $\beta \in C(\bar{\Omega},[0, \infty))$ and consider the equation

$$
\begin{aligned}
\partial_{t}^{2} u+\beta \partial_{t} u-\Delta u & =0 \text { in } \Omega \\
u & =0 \text { on } \partial \Omega .
\end{aligned}
$$

in the space $X=H_{0}^{1}(\Omega) \times L^{2}(\Omega)$. System (24) is equivalent to the system

$$
\begin{gathered}
\partial_{t} U=C U, \\
C=\left[\begin{array}{cc}
0 & I \\
-\Delta & -\beta I
\end{array}\right], U=\left[\begin{array}{c}
u \\
\partial_{t} u
\end{array}\right] .
\end{gathered}
$$


The operator $e^{C t}$ is a group on $\mathbb{R}$ and corresponds to the dynamical system generated by (25). If $\beta(x) \geq \delta>0$, then it is not difficult to show that the spectral radius of $e^{C}$ is $<1$. From Theorem 5.2, the origin $(0,0)$ is the compact global attractor for the dynamical system $C^{0}$-group $, e^{C t}, t \in \mathbb{R}$.

If there is an $x_{0} \in \Omega$ for which $\beta\left(x_{0}\right)>0$, it was shown by Iwasaki (1969), Dafermos (1978) that each solution of (24) approaches zero as $t \rightarrow \infty$. Furthermore, Dafermos (1978) demonstrated that there was a damping $\beta$ for which this approach to zero was not exponential. In such a case, the radius of the essential spectrum of $e^{C}$ is one. From Theorem 5.2, we have the following

Theorem 10.1. If $\beta(x) \geq \delta>0$ for $x \in \bar{\Omega}$, then $(0,0)$ is the compact global attractor of (25) and attracts compact sets of $X$.

There exists a $\beta$ such that the dynamical system defined by (25) has the property that $(0,0)$ is the maximal compact invariant set, $(0,0)$ is stable and attracts compacts of $X$, positive orbits of bounded sets are bounded and, finally, $(0,0)$ is not an attractor; that is, does not attract bounded sets.

It is very difficult to discuss perturbations, especially nonlinear ones, of (25) if $(0,0)$ is not the compact global attractor. If $(0,0)$ is the compact global attractor, then there are $K>0, \alpha>0$ such that

$$
\left|e^{C t}\right|_{\mathcal{L}(X, X)} \leq K e^{-\alpha t}, \quad t \geq 0 .
$$

Thus, it is important to determine conditions on $\beta$ which will ensure that (26) is satisfied. The definitive statement is due to Bardos et al. (1992).

Theorem 10.2. The point $(0,0)$ is the compact global attractor for (25) if and only if every light ray of the equation

$$
\partial_{t}^{2} u-\Delta u=0
$$

intersects the support $\omega$ of $\beta$.

In particular, this is true if either $\omega \supset \partial \Omega$ or, if $n=1$ and there is an $x_{0} \in \Omega$ for which $\beta\left(x_{0}\right)>0$.

Given a specific domain $\Omega$, it would be interesting to characterize the types of $\beta$ for which the conclusions of Theorem 10.2 are valid. How much of the boundary must the support of $\beta$ cover? Can one choose $\beta$ so that it intersects the boundary at a very small set and has special properties in $\Omega$ ? Some interesting examples are in Chen et al. (1991), Haraux (1989).

\subsection{Constant linearly damped nonlinear wave equation}

Let $\Omega$ be as above, $\beta>0$ a constant, $f: u \in \mathbb{R} \mapsto f(u) \in \mathbb{R}$ is a $C^{1}$ function and there exist positive constants $c, \gamma$ such that

$$
\begin{aligned}
& \left|f^{\prime}(u)\right| \leq c e^{|u|^{\gamma}}, \quad 0 \leq \gamma<2, n=2, \\
& \left|f^{\prime}(u)\right| \leq c\left(1+|u|^{\gamma}\right), \quad 0 \leq \gamma \leq 2, n=3 .
\end{aligned}
$$


Consider the linearly damped nonlinear wave equation

$$
\begin{aligned}
\partial_{t}^{2} u+\beta \partial_{t} u-\Delta u & =f(u) \text { in } \Omega \\
u & =0 \text { on } \partial \Omega .
\end{aligned}
$$

System (28) is equivalent to the system

$$
\begin{gathered}
\partial_{t} U=C U+\tilde{f}(U), \\
C=\left[\begin{array}{cc}
0 & I \\
-\Delta & -\beta I
\end{array}\right], \quad \tilde{f}(U)=\left[\begin{array}{c}
0 \\
f(u)
\end{array}\right], \quad U=\left[\begin{array}{c}
u \\
\partial_{t} u
\end{array}\right] .
\end{gathered}
$$

If we choose initial data $U_{0}=(\varphi, \psi) \in X \equiv H_{0}^{1}(\Omega) \times L^{2}(\Omega)$, then $U$ is a (mild) solution of (29) if it satisfies the integral equation

$$
U(t)=e^{C t} U_{0}+\int_{0}^{t} e^{C(t-s)} \tilde{f}(U(s)) d s, \quad t \geq 0 .
$$

It is possible to show

Lemma 10.3. If $f$ satisfies $(27)$, then $f: H_{0}^{1}(\Omega) \rightarrow L^{2}(\Omega)$ (and, therefore, $\tilde{f}: X \rightarrow$ $X)$ is a $C^{1}$-function which takes bounded sets into bounded sets.

The function $\tilde{f}$ is compact if $n=1,2$ and also if $n=3$ and $\gamma<2$.

From Lemma 10.3, one can use (30) and the contraction mapping theorem to show that, for any $(\varphi, \psi) \in X$, there is a solution $T_{f}(t)(\varphi \cdot \psi) \in X$ with initial value $(\varphi, \psi)$ at $t=0$ defined on a maximal interval $\left[0, t_{(\varphi, \psi)}\right)$; that is, $(29)$ defines a local dynamical system $T_{f}$ on $X$.

Since $\beta>0$ is a constant, we know from Theorem 10.1 that the estimate (26) holds. This is important for

Lemma 10.4. Let $T_{f}$ be the local dynamical system defined by (28). Suppose that $n=1,2$ or $n=3$ with $\gamma<2$. If $B$ is a closed bounded set of $X$ (considered as a metric space with the metric induced by $X$ ) such that $T_{f}(t) B \subset B$ for $t \geq 0$, then $T_{f}(t) \mid B, t \geq 0$, is a dynamical system on $B$ and it is asymptotically smooth

Proof. With $B$ as in the statement of the lemma, it is clear that $T_{f}(t) \mid B, t \geq 0$, is a dynamical system on $B$. Furthermore, from (30), (26) and Lemma 10.3, $T_{f}(t)$ is the sum of an operator which is completely continuous for each $t \geq 0$ and a linear operator that approaches zero exponentially as $t \rightarrow \infty$; that is, satisfies (2). We have noted that this implies that $T_{f}(t) \mid B, t \geq 0$, is asymptotically smooth on $B$.

From Theorem 5.7, to prove the existence of a compact global attractor, we need to know that $T_{f}$ is a dynamical system on all of $X$, is point dissipative and, for any bounded set $B \subset X$, there is a $t_{0}>0$ such that $\gamma^{+}\left(T_{f}\left(t_{0}\right) B\right)$ is bounded.

With a dissipative condition on $f$, we easily satisfy all of these requirements. 
The energy functional $V: X \rightarrow \mathbb{R}$ for (28) (and (29)) is

$$
V(\varphi, \psi)=\int_{\Omega}\left[\frac{1}{2}|\nabla \varphi(x)|^{2}+\frac{1}{2}|\psi(x)|^{2}-F(\varphi(x))\right] d x
$$

where $F(u)=\int_{0}^{u} f(s) d s$.

If $\left(u(t, x), \partial_{t} u(t, x)\right)$ is a solution of $(28)$, then, on a dense set of initial data, a simple computation shows that

$$
\dot{V}\left(u(t, \cdot), \partial_{t} u(t, \cdot)\right)=-\int_{\Omega} \beta\left|\partial_{t} u(t, x)\right|^{2} d x \leq 0 .
$$

This implies that $V\left(u\left(t, \cdot, \partial_{t} u(t, \cdot)\right)\right.$ is a nonincreasing function of $t$ for all solutions of (28).

Let us now impose the dissipative condition

$$
\limsup _{|u| \rightarrow \infty} \frac{f(u)}{u} \leq \lambda_{1}
$$

where $\lambda_{1}$ is the first eigenvalue of $-\Delta$ with homogeneous Dirichlet boundary conditions.

Theorem 10.5. If the conditions (32), (27) with $\gamma<2$ for $n=3$ are satisfied, then $T_{f}(t)$ is a dynamical system on $X$, the compact global attractor $\mathcal{A}_{f}$ exists and $T_{f}$ is a gradient system.

If $n=1$, then, for any $(\varphi, \psi) \in X, \omega(\varphi, \psi)$ is a singleton. If $n \geq 1$ and the function $f$ is analytic, then $\omega(\varphi, \psi)$ is a singleton.

Proof. From (32), one can deduce that $V(\varphi, \psi) \rightarrow \infty$ as $|(\varphi, \psi)| \rightarrow \infty$. Since $V$ is nonincreasing along solutions of (28), this implies that, for any bounded set $B \subset X$, $\gamma^{+}(B)$ exists and is bounded. Therefore, (28) defines a dynamical system $T_{f}$ on $X$. From Lemma $10.4, T_{f}$ is asymptotically smooth. As a consequence, $\omega(\varphi, \psi)$ is a compact invariant set. Since $V\left(u(t, \cdot), \partial_{t} u(t, \cdot)\right)$ is nonincreasing in $t, V$ must be constant on any $\omega$-limit set since such a set is invariant. From (31), elements of $\omega(\varphi, \psi)$ must be of the form $(\varphi, 0)$ with $-\Delta \psi=f(\psi$ in $\Omega, \psi=0$ on $\partial \Omega$; that is, the set of equilibria. A simmple variational argument shows that the set of equilibria is bounded and, thus, $T_{f}$ is point dissipative. Theorem 5.7 completes the proof of the existence of the compact global attractor.

The statement about $n=1$ is due to Hale and Raugel (1993) and is the consequence of an eigenvalue computation showing that the linear variational equation about an equilibrium can never have the eigenvalue zero with multiplicity greater that one.

The last statement about convergence to a singleton if $f$ is analytic is due to Haraux and Jendoubi (1999). This uses a more sophisticated technique as in the proof of Theorem 9.2. 
Theorem 10.5 as stated is due to Hale and Raugel (1993). Their proof is exactly the same as the one given by Hale (1983) in which a stronger hypothesis on $f$ was imposed in the case $n=2$. Haraux (1985) proved the above theorem with the same hypotheses as in Hale (1983) except that the domain was more regular and he made use of the fact that he could prove that the solutions belong to a more regular space. This type of proof will not work for the case of a domain for which the boundary is only Lipschitzian.

To the author's knowledge, Babin and Vishik (1983) were the first to discuss attractors for (33), but the definition was not the same as the one that we are using. They were able to prove that the set $\mathcal{A}(T)$ was an attractor in the sense that it would attract bounded sets of the space $Y=\left(H^{2}(\Omega) \cap H_{0}^{1}(\Omega)\right) \times H^{1}(0)$; that is, special compact sets of $X$. This is analogous to the result of Hale and Meyer (1967) on the attractor for a linear neutral functional differential equation with a stable $D$ operator. We have discussed this in some detail in the Appendix and noted that later work showing that the attractor for neutral equations was an attractor in the sense of these notes led to asymptotically smooth dynamical systems, a concept which turned out to be very appropriate for (33) as well as other partial differential equations of hyperbolic type.

For $\gamma=2, n=3$, the proof of Theorem 10.5 will not work since the map $\tilde{f}$ : $H_{0}^{1}(\Omega) \rightarrow L^{2}(\Omega)$ is a bounded map but not compact. In this case, Arrieta et al. (1992) proved that the compact global attractor exists. Their proof used a nonlinear variation of constants formula and a special type of decomposition of the function $f$. The decomposition consisted in writing $f=f_{0}+f_{1}$ with both $f_{0}$ and $f_{1}$ satisfying the dissipative condition (32), $f_{1}$ having growth rate (27) with $\gamma<2$ and $f_{0}$ satisfying the condition $f_{0}(0)=0, u f_{0}(u) \leq 0$ for all $u$. The function $f_{0}$ has the property that the origin is the compact global attractor for the dynamical system $T_{f_{0}}$ defined by (28) with $f$ replaced by $f_{0}$. Furthermore, for any bounded set $B \subset X$, the origin attracts $B$ under $T_{f_{0}}(t)$ at an exponential rate. Treating $f=f_{0}+f_{1}$ as a nonhomogeneous perturbation of $f_{0}$, the nonlinear variation of constants formula permits one to write the solution with initial value $(\varphi, \psi)$ as the sum of the solution of (28) with $f$ replaced by $f_{0}$ and with initial value $(\varphi, \psi)$ and a part which vanishes at $t=0$. It is then shown that the mapping $T_{f}(t)-T_{f_{0}}(t)$ is completely continuous for $t \geq 0$. The proof of this fact used some regularity of $T_{f}(t)(\varphi, \psi)-T_{f_{0}}(t)(\varphi, \psi)$ in the spatial variables. This shows that $T_{f}$ is asymptotically smooth. Theorem 5.7 completes the proof of the existence of the compact global attractor.

Babin and Vishik (1992) also proved Theorem 10.5 but imposed more restrictions on the nonlinear function $f$. With the decomposition of Arrieta et al. (1992), Raugel has pointed out to the author that the proof of Babin and Vishik (1992) can be used to obtain the general result. Another proof using functionals has been given by Ball (2003). 


\subsection{Local linearly damped nonlinear wave equation}

In this subsection, we assume the same conditions on $f$ as above; namely, (27) and (32), but we allow the linear damping to be a continuous nonnegative function of $x \in \Omega$.

Consider the equation

$$
\begin{aligned}
\partial_{t}^{2} u+\beta(x) \partial_{t} u-\Delta u & =f(u) \text { in } \Omega \\
u & =0 \text { on } \partial \Omega .
\end{aligned}
$$

If $C_{\beta}$ is defined as

$$
C_{\beta}=\left[\begin{array}{cc}
0 & I \\
-\Delta & -\beta(\cdot) I
\end{array}\right],
$$

then we assume that there are positive constants $K, \alpha$ such that

$$
\left|e^{C_{\beta} t}\right|_{\mathcal{L}(X, X)} \leq K e^{-\alpha t}, t \geq 0 .
$$

Theorem 10.2 gives necessary and sufficient conditions for (34) to hold.

The following result is due to Hale and Raugel (1993).

Theorem 10.6. If $f$ satisfies (27) with $\gamma<2$ for $n=3$ and (32) and (34) is satisfied, then (33) defines a dynamical system $T_{\beta, f}$ on $X$ and the minimal global attractor $\mathcal{A}_{\beta, f}$ exists; that is, $\mathcal{A}_{\beta, f}$ is invariant, attracts bounded sets of $X$ and is minimal with respect to this property.

If, in addition, $T_{\beta, f}$ is point dissipative, then $\mathcal{A}_{\beta, f}$ is the compact global attractor.

If either $n=1$ and there is an $x_{0} \in \Omega$ such that $\beta\left(x_{0}\right)>0$ or the support $\omega$ of $\beta$ contains the boundary of $\Omega$, then (33) is a gradient system and $\mathcal{A}_{\beta, f}$ is the compact global attractor.

If $n=1$, for any $(\varphi, \psi) \in X, \omega(\varphi, \psi)$ is a singleton.

The proof of the existence of the minimal global attractor is the same as for the case with $\beta>0$ a constant. In fact, it is easy to see that the argument for $\beta>0$ a constant shows that (33) defines a dynamical system $T_{\beta, f}$ on $X$, is asymptotically smooth and $\gamma^{+}(B)$ is bounded if $B \subset X$ is bounded. This implies that $\omega(B)$ is a compact invariant set. If we define

$$
\mathcal{A}_{\beta, f}=\operatorname{Cl}\{\omega(B): B \subset X \text { is bounded }\}
$$

then $\mathcal{A}_{\beta, f}$ is the minimal global attractor. If, in addition, $T_{\beta, f}$ is point dissipative, then $\mathcal{A}_{\beta, f}$ is the compact global attractor. This proves the first two parts of the theorem.

To prove the next part, notice that (28) implies that any globally defined solution of (33) must be a solution of the equation

$$
\begin{aligned}
\partial_{t}^{2} v-\Delta v & =f(v) \text { in } \Omega \backslash \omega \\
\partial_{t} v & =0 \text { in } \omega \\
v & =0 \text { in } \partial \Omega .
\end{aligned}
$$


The next step, which is the most difficult, is to show that the only solutions of (35) are equilibria. The proof, under the above assumptions on $\beta$, can be found in Hale and Raugel (1993). We only remark that it uses some regularity results to reduce the problem to the unique continuation property for the solutions of linear wave equations.

To prove the last statement concerning $n=1$, one shows first that, if an equilibrium is not hyperbolic, then 0 is a simple eigenvalue of the linear variational equation and is the only eigenvalue on the imaginary axis. The convergence to a singleton is now a consequence of Hale and Raugel (1992).

It is not known if the conclusions of Theorem 10.6 remain true if it is assumed only that inequality (34) is satisfied in the case $n \geq 2$; that is, is the condition $\omega \supset \partial \Omega$ necessary in Theorem 10.6?

\subsection{Generically Morse-Smale}

The author was informed by G. Raugel that the function $f$ in Theorem 9.5 serves as a counterexample to generic transversality of stable and unstable manifolds of hyperbolic equilibria of (28) in the class of $C^{1}$-functions $f$ which are spatially independent.

On the other hand, Brunovský and Raugel (2003) have given general results on generic transversality of stable and unstable manifolds for (32) in an appropriate class of functions $f$ which are spatially dependent. These results generalize Theorem 9.4 for parabolic equations.

\section{Appendix}

\section{A. Functional Differential Equations}

Retarded functional differential equations with finite delay (RFDE) and a special class of neutral functional differential equations with finite delay (NFDE) seem to have been the first evolutionary equations in an infinite dimensional nonlocally compact space for which a qualitative theory was developed. The detailed study of these equations played an important role in the present theory of the qualitative theory of quasilinear equations of parabolic and hyperbolic type. It is for this reason, as well as the fact that most researchers are not familiar with these equations, that we present in this appendix the definitions and some of the important properties of the dynamical system generated by these equations. The reader may consult Hale and Verduyn Lunel (1993) and Diekmann et al. (1995) for other aspects of the theory as well as further references. 


\section{A.1. RFDE}

For a given $\delta \geq 0$, let $C \equiv C\left([-\delta, 0], \mathbb{R}^{n}\right)$ be the space of continuous functions from $[-\delta, 0]$ to $\mathbb{R}^{n}$ with the sup norm $|\cdot| \equiv|\cdot|_{C}$. If $\delta=0$, we understand this space to be just $\mathbb{R}^{n}$. For any $\alpha \geq 0$, for any function $x:[-\delta, \alpha] \rightarrow \mathbb{R}^{n}$ and, for any $t \in[0, \alpha]$, we let $x_{t}$ denote the function from $[-\delta, 0]$ to $\mathbb{R}^{n}$ defined by $x_{t}(\theta)=x(t+\theta), \theta \in[-\delta, 0]$.

Suppose that $f: C \rightarrow \mathbb{R}^{n}$ is a $C^{r}$-function, $r \geq 1$, and is a bounded map; that is, takes bounded sets into bounded sets. An autonomous retarded functional differential equation (RFDE) with finite delay is a relation

$$
\dot{x}(t)=f\left(x_{t}\right)
$$

where $\dot{x}(t)$ is the right hand derivative of $x(t)$ at $t$.

For a given function $\varphi \in C$, we say that $x(t, \varphi)$ is a solution of (36) on the interval $\left[0, \alpha_{\varphi}\right), \alpha_{\varphi}>0$, with initial value $\varphi$ at $t=0$ if $x(t, \varphi)$ is defined on $\left[-\delta, \alpha_{\varphi}\right)$, $x_{t}(\cdot, \varphi) \in C$ for $t \in\left[0, \alpha_{\varphi}\right), x_{0}(\cdot, \varphi)=\varphi$ and $x(t, \varphi)$ satisfies $(36)$ on the interval $\left[0, \alpha_{\varphi}\right)$. If a solution of (36) exists, then, for each $t \in\left[0, \alpha_{\varphi}\right)$, it must satisfy the following equation

$$
\begin{aligned}
x_{0}(\cdot, \varphi) & =\varphi \\
x(t, \varphi) & =\varphi(0)+\int_{0}^{t} f\left(x_{s}(\cdot, \varphi)\right) d s, \quad t \in\left[0, \alpha_{\varphi}\right) .
\end{aligned}
$$

From (37), it is clear that a solution $x(t, \varphi)$ must be continuously differentiable for $t \in\left(0, \alpha_{\varphi}\right)$ with a right hand derivative at $t=0$. We remark that a solution $x(t, \varphi)$ will be continuously differentiable at $t=0$ if and only if the initial data $\varphi$ is continuously differentiable at $\theta=0$ and $d \varphi(0) / d \theta=f(\varphi)$.

The proof of the following result may be easily given by using the contraction mapping principle in (37)

Theorem A.1. For any $\varphi \in C$, there is a unique solution $x(t, \varphi)$ defined on a maximal interval $\left[-\delta, \alpha_{\varphi}\right), x(t, \varphi)$ is continuous in $(t, \varphi)$ and $C^{r}$ in $\varphi$ for all $t \in$ $\left[-\delta, \alpha_{\varphi}\right), \varphi \in C$. Also, for $k \leq r+1, x(t, \varphi)$ is $C^{k}$ in $t$ if $t \in\left(k \delta, \alpha_{\varphi}\right)$.

If $\alpha_{\varphi}<\infty$, then $\left|x_{t}(\cdot, \varphi)\right| \rightarrow \infty$ as $t \rightarrow \alpha_{\varphi}^{-}$

If we define $T(t) \varphi \equiv x_{t}(\cdot, \varphi)$ for $t \in\left[0, \alpha_{\varphi}\right)$, then we obtain a local dynamical system on $C$. If all solutions exist on $[-\delta, \infty)$, then $T: \mathbb{R}^{+} \times C \rightarrow C$ is a dynamical system on $C$.

A mapping $M: C \rightarrow C$ is said to be conditionally completely continuous if, for any bounded $B \subset C$ for which $M B$ is bounded, the closure of $M B$ is compact. An application of the Arzelá-Ascoli theorem and Theorem 5.1 yield the following result.

Theorem A.2. The dynamical system $T(t), t \geq 0$, defined by (36) is conditionally completely continuous for $t \geq \delta$. If $T(t)$ is a bounded map for each $t \geq 0, T(t), t \geq 0$, is completely continuous for $t \geq \delta$.

If $T(t)$ is point dissipative, then the compact global attractor exists. 
For a complete discussion of RFDE, it also is necessary to understand the structure of $T(t)$ on the interval $[0, \delta]$. This is a consequence of discussing the solution of the equation $x(t)=0$ considered as a dynamical system $T_{0}(t), t \geq 0$, on the space $C_{0}=\{\psi \in C: \psi(0)=0\}$. It is clear that, for any $\psi \in C_{0}$,

$$
\begin{aligned}
& \left(T_{0}(t) \psi\right)(0)=0, \quad t \geq 0, \\
& \left(T_{0}(t) \psi\right)(\theta)=\psi(t+\theta), \quad t+\theta \in[-\delta, 0) .
\end{aligned}
$$

Let us rewrite (37) as

$$
\begin{aligned}
& (T(t) \varphi)(0)=0+\varphi(0)+\int_{0}^{t} f(T(s) \varphi) d s, \quad \geq 0, \\
& (T(t) \varphi)(\theta)=[\varphi(t+\theta)-\varphi(0)]+\varphi(0), \quad t+\theta \in[-\delta, 0) .
\end{aligned}
$$

Since the function $\varphi-\varphi(0)$ is an element of $C_{0}$, the solution of $x(t)=0$ through $\varphi-\varphi(0)$ is given by $T_{0}(t)[\varphi-\varphi(0)]$. From relation (38), we observe that $T(t)$ is the sum of $T_{0}(t)[\varphi-\varphi(0]$ and a conditionally completely continuous operator. We can also introduce an equivalent norm in $C$ such that $T_{0}(t)[\varphi-\varphi(0)]$ is a strict contraction for each $t>0$. As a consequence, we have

Theorem A.3. For each $t \geq 0$,

$$
\begin{aligned}
& T(t)=T_{0}(t)[\varphi-\varphi(0)]+U(t) \text { is a conditional } \alpha \text {-contraction, } \\
& U(t) \text { is conditionally completely continuous, } \\
& T_{0}(t) \text { is the dynamical system on } C_{0} \text { defined by } x(t)=0 .
\end{aligned}
$$

If $T(t)$ is a bounded map for each $t \geq 0$, then it is an $\alpha$-contraction for all $t \geq 0$.

For equation (36) with $f(t, \varphi), f(t+\tau, \varphi)=f(t, \varphi)$, we can define the period map $P$ on $C$ which takes the initial value at time zero to the solution in $C$ at time $\tau$. This map also will be an $\alpha$-contraction for an appropriate norm in $C$. There exists an integer $k$ such that $P^{k}$ is completely continuous. If $P$ is point dissipative, then Theorem 5.1 implies that there is the compact global attractor $\mathcal{A}(P)$ of $P$. In particular, $\mathcal{A}(P)$ attracts compact sets. From these remarks and Theorem 5.6, we see that point dissipativeness of $P$ will imply a fixed point of $P$. Such a fixed point corresponds to a $\tau$-periodic solution of the equation. Previous to this remark, researchers had been able to obtain $\tau$-periodic solutions only for $\tau \geq \delta$ since they were making use only of the fact that $P$ was completely continuous and not using the structure of the dynamical system on the interval $[0, \tau)$.

\section{A.2. NFDE}

We use the same notation as for RFDE. 
Suppose that $D: C \rightarrow \mathbb{R}^{n}$ is a continuous linear operator, which is atomic at zero; that is,

$$
D \varphi=\varphi(0)-\int_{-\delta}^{0} d \mu(\theta), \varphi(\theta)
$$

wnere $\mu(\theta)$ is an $n \times n$ matrix of bounded variation and there is a continuous nondecreasing function $\gamma(s), s \in[-\delta, 0], \gamma(0)=0$ such that, for any $s>0$,

$$
\operatorname{Var}_{[-s, 0]} \mu \leq \gamma(s) .
$$

Suppose that $f: C \rightarrow \mathbb{R}^{n}$ is a $C^{r}$-function, $r \geq 1$, and is a bounded map. A neutral functional differential equation (NFDE) is a relation

$$
\frac{d}{d t} D x_{t}=f\left(x_{t}\right)
$$

where $\frac{d}{d t}$ is the right hand derivative at $t$.

Note that an RFDE is a special case of (41) with $D \varphi=\varphi(0)$.

For a given function $\varphi \in C$, we say that $x(t, \varphi)$ is a solution of (41) on the interval $\left[0, \alpha_{\varphi}\right), \alpha_{\varphi}>0$, with initial value $\varphi$ at $t=0$ if $D x_{t}(\cdot, \varphi)$ is defined on $\left[-\delta, \alpha_{\varphi}\right), x_{t}(\cdot, \varphi) \in C$ for $t \in\left[0, \alpha_{\varphi}\right), x_{0}(\cdot, \varphi)=\varphi$ and $x(t, \varphi)$ satisfies (41) on the interval $\left[0, \alpha_{\varphi}\right)$.

If a solution of (41) exists, then it must satisfy the following equation

$$
\begin{aligned}
x_{0}(\cdot, \varphi) & =\varphi \\
D x_{t}(\cdot, \varphi) & =D \varphi+\int_{0}^{t} f\left(x_{s}(\cdot, \varphi)\right) d s, \quad t \in\left[0, \alpha_{\varphi}\right) .
\end{aligned}
$$

From (42), it is clear that each solution $x(t, \varphi)$ of (41) has the property that $D x_{t}(\cdot, \varphi)$ must be continuously differentiable for $t \in\left(0, \alpha_{\varphi}\right)$ with a right hand derivative at $t=0$.

We emphasize that we do not require that the function $x(t, \varphi)$ have a right hand derivative, but only that the function $D x_{t}(\cdot, \varphi)$ has a right hand derivative.

If a solution of (41) is continuously differentiable, then it satisfies the relation $D \dot{x}_{t}=f\left(x_{t}\right)$, an equation in which the derivative occurs with delayed arguments. Our definition of NFDE is a special case of a more general class of FDE for which the derivatives occur with delayed arguments in more general form (see, for example, Kolmanovskiı̌ and Myshkis (1992)). However, (40) occurs frequently in the applications and, for such systems, we can develop a qualitative theory which is as general as the one for RFDE. Hale and Meyer (1967) were the first to consider NFDE in the form (41) where it is not required for a solution $x(t)$ to be $C^{1}$, but that $D x_{t}$ be $C^{1}$.

It can be shown that equations of the type (41) are very closely related to weak solutions of undamped wave equations in one space dimension with linear or nonlinear boundary conditions (see Abolinya and Myškis (1960), Brayton (1966), Cooke and Krumme (1968)). 
Theorem A.4. If $f \in C^{r}\left(C, \mathbb{R}^{n}\right), r \geq 1$ (resp. analytic), then, for any $\varphi \in C$, there is a unique solution $x(t, \varphi)$ of (26) on a maximal interval of existence $\left[-\delta, \alpha_{\varphi}\right)$. If $\alpha_{\varphi}<\infty$, then $\left|x_{t}(\cdot, \varphi)\right| \rightarrow \infty$ as $t \rightarrow \alpha_{\varphi}$. Furthermore, $x_{t}(\cdot, \varphi)$ is continuous in $t, \varphi$ and is $C^{r}$ in $\varphi$ (resp. analytic).

The proof of Theorem A.4 follows in spirit the proof of Theorem A.1 except applied to the integral equation (42) written in the form

$$
\begin{aligned}
x_{0}(\cdot, \varphi) & =\varphi \\
x(t) & =\int_{-\delta}^{0}[d \mu(\theta)] x(t+\theta)+D \varphi+\int_{0}^{t} f\left(x_{s}(\cdot, \varphi)\right) d s, \quad t \in\left[0, \alpha_{\varphi}\right) .
\end{aligned}
$$

In this case, it does not seem possible to use the contraction principle to get a fixed point of a map defined by the right hand side of the equalities in this equation. However, it is possible to show that, on an appropriate class of functions on an interval $[-\delta, a]$ with $a$ small, one has an $\alpha$-contraction. A fixed point theorem for $\alpha$-contractions gives existence. The maximal interval of existence is obtained by invoking Zorn's lemma in the usual way. The uniqueness and smoothness properties of the solution in $\varphi$ require some special arguments (for details, see Hale and Verduyn Lunel (1993)).

Let $T(t) \varphi \equiv T_{D, f}(t) \varphi=x_{t}(\cdot, \varphi)$ for $t \in\left[0, \alpha_{\varphi}\right)$. If we assume that all solutions of (41) are defined for $t \in \mathbb{R}^{+}$, then the family of maps $\left\{T_{D, f}(t): C \rightarrow C, t \geq 0\right\}$ is a $C^{r}$-semigroup on $C$ if $f$ is $C^{r}$ and analytic if $f$ is analytic. We will assume in the following that all solutions of (41) are defined for $t \geq 0$.

Our next objective is to obtain an important qualitative property of the dynamical system generated by solutions of (41) which, for RFDE, reduces to Theorem A.3. Historically, the observation for RFDE was noted after the result had been proved for NFDE.

To do this, we make the assumption that the matrix function $\mu(\theta)$ of bounded variation in (39) has no singular part; that is,

$$
\begin{aligned}
& D \varphi=D_{0} \varphi-\int_{-\delta}^{0} B(\theta) \varphi(\theta) d \theta \\
& D_{0} \varphi=\varphi(0)-\Sigma_{j=1}^{\infty} B_{j} \varphi\left(-\delta_{j}\right), \quad \delta_{j}>0,1 \leq j, \\
& \Sigma_{j=1}^{\infty}\left|B_{j}\right|<\infty, \quad \int_{-\delta}^{0}|B(\theta)| d \theta<\infty .
\end{aligned}
$$

We say that a linear operator $D: C \rightarrow \mathbb{R}^{n}$ is exponentially stable if the zero solution of the equation $D y_{t}=0$ is exponentially stable; that is, there are positive constants $k, \alpha$ such that, for any $\psi \in C$ with $D \psi=0$, the solution $y(t, \varphi)$ of $D y_{t}=0$ with $y_{0}(\cdot, \psi)=\psi$ satisfies $\left|y_{t}(\cdot, \psi)\right| \leq k \exp (-\alpha t)|\psi|$ for $t \geq 0$.

Theorem A.5. Let $T(t) \equiv T_{D, f}(t), t \geq 0$, be the dynamical system defined by (41) with $D$ satisfying (43) and let $T_{D_{0}}$ be the dynamical system on $C_{D_{0}}=\left\{\varphi \in C: D_{0} \varphi=\right.$ 
$0\}$ generated by the difference equation $D_{0} y_{t}=0$. Then there is a matrix function $\Psi_{D_{0}}: C \rightarrow C_{D_{0}}$ such that

$$
\begin{aligned}
& T_{D, f}(t)=T_{D_{0}}(t) \Psi_{D_{0}}+U_{D, f}(t), \\
& U_{D, f}(t) \text { conditionally completely continuous for } t \geq 0,
\end{aligned}
$$

If, in addition, the operator $D_{0}$ is exponentially stable, then there is an equivalent norm in $C$ such that, for each $t>0, T_{D, f}(t)$ is a conditional $\alpha$-contraction.

We present a sketch of the ideas in the proof. The solutions of (41) coincide with the solutions of (42). The first step is to show that there exists a matrix function $\Phi_{D_{0}}=\left(\varphi_{1}^{D_{0}}, \ldots, \varphi_{n}^{D_{0}}\right), D \Phi_{D_{0}}=I$, where each $\varphi_{j}^{D_{0}} \in C$ and to define $\Psi_{D_{0}}=I-$ $\Phi_{D_{0}} D_{0}$. Then $\Psi_{D_{0}}: C \rightarrow C_{D_{0}}$, If we let $U(t) \varphi \equiv U_{D, f}(t) \varphi=T_{D, f}(t) \varphi-T_{D_{0}}(t) \Psi_{D_{0}} \varphi$ and $T(t) \varphi=T_{D, f}(t) \varphi$, then, for $t \geq 0$,

$$
D_{0}(U(t) \varphi)=\int_{t-\delta}^{t} B(s-t)(T(s) \varphi)(0) d s+\int_{0}^{t} f(T(s) \varphi) d s
$$

and $U(0) \varphi=\Phi_{D_{0}} D_{0} \varphi$, which varies over a finite dimensional subspace as $\varphi$ varies over $C$. One now uses some nontrivial estimates to show that $U(t)$ is conditionally completely continuous. This gives the decomposition (44). If $D_{0}$ is stable, then one can find an equivalent norm in $C$ such that $T_{D, f}(t)$ is an $\alpha$-contraction.

This result is essentially contained in Hale (1970) and made extensive use of Cruz and Hale (1969), (1970), (1971) (see, also Henry (1974)).

Knowing that a NFDE has the representation in Theorem A.5 led to the general class of asymptotically smooth dynamical systems of Section 5 introduced by Hale et al. (1972). In fact, if $D_{0}$ is exponentially stable, then the dynamical system $T_{D, f}$ is asymptotically smooth. The asymptotically smooth dynamical systems were introduced with the hope the one also could discuss compact global attractors for certain types of partial differential equations which exhibited hyperbolic structure. The concept has turned out to very appropriate for many problems, one of which was mentioned in Section 10.

If $T_{D, f}(t)$ is a linear operator for each $t$ (that is, the original equation (41) is linear), then the representation (44) shows that

$$
r\left(\sigma_{\text {ess }}\left(T_{D, f}(1)\right)\right)=r\left(\sigma_{\text {ess }}\left(T_{D_{0}}(1)\right)\right) .
$$

As a consequence, if $D_{0}$ is exponentially stable and $T_{D, f}$ is point dissipative, then it is not difficult to show that there are positive constants $k, \alpha$ such that

$$
\left\|T_{D, f}(t)\right\|_{\mathcal{L}(C, C)} \leq k e^{-\alpha t}, \quad t \geq 0 .
$$

Let $A_{D, f}$ be the infinitesimal generator of $T_{D, f}(t), t \geq 0$; that is,

$$
\mathcal{D}\left(A_{D, f}\right)=\left\{\varphi \in C: \lim _{t \rightarrow 0^{+}} \frac{1}{t}\left[T_{D, f}(t) \varphi-\varphi\right] \text { exists }\right\}
$$


and $A_{D, f} \varphi$ is this limit. It is easy to show that

$$
\mathcal{D}\left(A_{D, f}\right)=\left\{\varphi \in C^{1}\left([-\delta, 0], \mathbb{R}^{n}\right): D \dot{\varphi}=f(\varphi)\right\}
$$

A simple computation shows that $A_{D, f}$ has compact resolvent with only point spectrum given by

$$
\sigma\left(A_{D, f}\right)=\left\{\lambda \in \mathbb{C}: \operatorname{det}\left[D e^{\lambda \cdot} I-f\left(e^{\lambda \cdot} I\right)\right]=0 .\right.
$$

It is to be expected that, if $\operatorname{Re}\left(\sigma\left(A_{D, f}\right)\right)<0$, then $T_{D, f}$ satisfies (45). Hale and Meyer (1967) discussed exactly this situation, but were unable to prove it. By using the inverse Laplace transform for $T_{D, f}(t) \varphi$ and integrating by parts they showed that

$$
\left|T_{D, f}(t) \varphi\right|_{C} \leq k e^{-\alpha t}|\varphi|_{\mathcal{D}_{D, f}}, \quad t \geq 0,
$$

where $|\varphi|_{\mathcal{D}_{D, f}}=\max \left\{|\varphi|_{C},|\dot{\varphi}|_{C}\right\}$ and $\varphi \in \mathcal{D}_{D, f}$.

Similar results hold for the dynamical system $T_{D_{0}}$ on $C_{D_{0}}$. The infinitesimal generator $A_{D_{0}}$ is given by $A_{D_{0}} \psi=\dot{\psi}$ for

$$
\psi \in \mathcal{D}\left(A_{D_{0}}\right) \equiv\left\{\psi \in C_{D_{0}} \cap C^{1}: D_{0} \dot{\psi}=0\right\}
$$

Also, $A_{D_{0}}$ has compact resolvent and

$$
\sigma\left(A_{D_{0}}\right)=\left\{\lambda \in \mathbb{C}: \operatorname{det} D e^{\lambda \cdot} I=0\right\} .
$$

Cruz and Hale (1969), (1970), (1971) were able to show that the estimate (45) was valid if $D_{0}$ was exponentially stable. However, this did not relate the spectrum of the generator of $T_{D_{0}}(t)$ to the spectrum of its generator $A_{D_{0}}$. In 1987, Henry (see also Greiner and Schwarz (1991)) proved the following result.

\section{Theorem A.6.}

$$
\sigma_{e s s}\left(T_{D, f}(t)\right)=\sigma_{e s s}\left(\left(T_{D_{0}}(t)\right)=\mathrm{Cl}_{\mathbb{C}} e^{\sigma\left(A_{D_{0}}\right) t}, \quad t>0 .\right.
$$

Example A.7. We give now an example of a NFDE showing that both situations in Theorem 5.2 can occur. Consider the scalar NFDE

$$
\frac{d}{d t}[x(t)-a x(t-1)]+c x(t)=0
$$

where $c, a$ are constants. In the notation for NFDE, we have $C=C([-1,0], \mathbb{R})$, $D \varphi \equiv D_{a} \varphi=\varphi(0)-a \varphi(-1), C_{D_{a}}=\{\varphi \in C: \varphi(0)-a \varphi(-1)=0\}, f(\varphi)=-c \varphi(0)$.

Let $T_{a, c}$ be the dynamical system on $\mathrm{C}$ generated by (46) and $T_{D_{a}}$ be the dynamical system on $C_{D_{a}}$ generated by

$$
D_{a}\left(y_{t}\right)=y(t)-a y(t-1)=0 .
$$


The infinitesimal generator $A_{a, c}$ of $T_{a, c}$ is defined on the domain

$$
\mathcal{D}\left(A_{a, c}\right)=\left\{\varphi \in C: \varphi \in C^{1}, \varphi^{\prime}(0)-a \varphi^{\prime}(-1)+c \varphi(0)=0\right\}
$$

and $A_{a, c} \varphi=\varphi^{\prime}$ if $\varphi \in \mathcal{D}\left(A_{a, c}\right)$. The operator $A_{a, c}$ has compact resolvent and only point spectrum which is given by the values $\lambda$ that satisfy the equation

$$
\lambda\left(1-a e^{-\lambda}\right)+c=0 .
$$

The operator $A_{a}$ has compact resolvent and only point spectrum which is given by the values $\lambda$ that satisfy the equation

$$
1-a e^{-\lambda}=0 .
$$

From Theorem A.6 (or direct calculation since the equations is so simple), we see that $\sigma\left(T_{D_{0}}(1)\right)=\sigma_{\text {ess }}\left(T_{D_{0}}(1)\right)$ lies on the circle in the complex plane with center zero and modulus $|a|$. As a consequence, $r\left(\sigma_{e s s}\left(T_{a, c}(t)\right)\right)=|a|$.

It is not difficult to prove that $\sigma\left(T_{a, c}(t)\right)$ is continuous in $a, c$. Also, for $|a| \leq 1$, it is easy to show that there are no solutions of (47) on the imaginary axis. For $a=0$, $\sigma\left(T_{0, c}(t)\right)=\left\{0, e^{-c t}\right\}$. As a consequence, if $c>0$, then $\left|\sigma\left(T_{a, c}(t)\right)\right| \leq 1$ and it is $<1$ if $|a|<1$. In this case, the origin is the compact global attractor.

If $|a|=1$, then $r\left(\sigma_{\text {ess }}\left(T_{a, c}(t)\right)\right)=1$. With more effort, one can show that the Laplace transform of any solution of (46) has an analytic extension to the closed right half plane $\{z \in \mathbb{C}: \operatorname{Re} z \geq 0\}$. This implies that the solution is integrable along the positive real axis and, hence, each solution tends to zero as $t \rightarrow \infty$; that is, $T_{a, c}$ is point dissipative. As a consequence of Theorem 5.2, the origin attracts compact sets, is stable and positive orbits of bounded sets are bounded, but the compact global attractor does not exist. For details of this argument, see Hale and Verduyn Lunel (2002).

\section{References}

Abolinya, V. È. and A. D. Myškis. 1960. Mixed problems for quasi-linear hyperbolic systems in the plane, Mat. Sb. (N.S.) 50 (92), 423-442. (Russian)

Andronov, A. A. and L. Pontryagin. 1937. Systèmes grossiers., Dok. Akad. Nauk SSSR 14, $247-250$.

Angenent, S. B. 1986. The Morse-Smale property for a semilinear parabolic equation, J. Differential Equations 62, 427-442.

Arnol'd, V. I. 1988. The branched covering $\mathbf{C P}^{2} \rightarrow S^{4}$, hyperbolicity and projective topology, Sibirsk. Mat. Zh. 29, 36-47, 237. (Russian)

Arrieta, J., A. N. Carvalho, and Jack K. Hale. 1992. A damped hyperbolic equation with critical exponent, Comm. Partial Differential Equations 17, 841-866.

Aubin, D. and A. Dahan Dalmedico. 2002. Writing the history of dynamical systems and chaos: longue durée and revolution, disciplines and cultures, Historia Math. 29, 273-339.

Babin, A. V. and M. I. Vishik. 1983. Regular attractors of semigroups and evolution equations, J. Math. Pures Appl. (9) 62, 441-491 (1984). 
1989. Uniform finite-parameter asymptotics of solutions of nonlinear evolutionary equations, J. Math. Pures Appl. (9) 68, 399-455 (1990).

1992. Attractors of evolution equations, Studies in Mathematics and its Applications, vol. 25, North-Holland Publishing Co., Amsterdam, ISBN 0-444-89004-1, Translated and revised from the 1989 Russian original by Babin.

Ball, J. M. 1997. Continuity properties and global attractors of generalized semiflows and the NavierStokes equations, J. Nonlinear Sci. 7, 475-502.

1998. Erratum: "Continuity properties and global attractors of generalized semiflows and the Navier-Stokes equations", J. Nonlinear Sci. 8, 233.

2003. Global attractors for damped semilinear wave equations, Disc. Cont. Dyn. Sys.

Bardos, C., G. Lebeau, and J. Rauch. 1992. Sharp sufficient conditions for the observation, control, and stabilization of waves from the boundary, SIAM J. Control Optim. 30, 1024-1065.

Bhatia, N. P. and G. P. Szegö. 1967. Dynamical systems: Stability theory and applications, Lecture Notes in Mathematics, No. 35, Springer-Verlag, Berlin.

Brayton, Robert K. 1966. Bifurcation of periodic solutions in a nonlinear difference-differential equations of neutral type, Quart. Appl. Math. 24, 215-224.

Billotti, J. E. and J. P. LaSalle. 1971. Dissipative periodic processes, Bull. Amer. Math. Soc. 77, $1082-1088$.

Brunovský, P. and S.-N. Chow. 1984. Generic properties of stationary state solutions of reactiondiffusion equations, J. Differential Equations 53, 1-23.

Brunovský, P. and P. Poláčik. 1997. The Morse-Smale structure of a generic reaction-diffusion equation in higher space dimension, J. Differential Equations 135, 129-181.

Brunovský, P. and G. Raugel. 2003. Genericity of Morse-Smale systems in linearly damped nonlinear wave equations (to appear).

Chen, G., S. A. Fulling, F. J. Narcowich, and S. Sun. 1991. Exponential decay of energy of evolution equations with locally distributed damping, SIAM J. Appl. Math. 51, 266-301.

Chicone, C. and Y. Latushkin. 1999. Evolution semigroups in dynamical systems and differential equations, Mathematical Surveys and Monographs, vol. 70, American Mathematical Society, Providence, RI, ISBN 0-8218-1185-1.

Cholewa, J. W. and J. K. Hale. 2000. Some counterexamples in dissipative systems, Dynam. Contin. Discrete Impuls. Systems 7, 159-176.

Conley, C. 1978. Isolated invariant sets and the Morse index, CBMS Regional Conference Series in Mathematics, vol. 38, American Mathematical Society, Providence, R.I., ISBN 0-8218-1688-8.

Cooke, Kenneth L. and David W. Krumme. 1968. Differential-difference equations and nonlinear initial-boundary value problems for linear hyperbolic partial differential equations, J. Math. Anal. Appl. 24, 372-387.

Cooperman, G. 1978. $\alpha$-condensing maps and dissipative systems, Brown University, Providence, Ph. D. Thesis.

Cruz, M. A. and J. K. Hale. 1969. Asymptotic behavior of neutral functional differential equations, Arch. Rational Mech. Anal. 34, 331-353.

1970. Stability of functional differential equations of neutral type, J. Differential Equations 7, 334-355. MR 41 \#2166

. 1971. Exponential estimates and the saddle point property for neutral functional differential equations, J. Math. Anal. Appl. 34, 267-288. 
Dafermos, C. M. 1978. Asymptotic behavior of solutions of evolution equations, Nonlinear Evolution Equations (Proc. Sympos., Univ. Wisconsin, Madison, Wis., 1977), Publ. Math. Res. Center Univ. Wisconsin, vol. 40, Academic Press, New York, pp. 103-123.

Diekmann, O., S. A. van Gils, S. M. Verduyn Lunel, and H.-O. Walther. 1995. Delay equations, Applied Mathematical Sciences, vol. 110, Springer-Verlag, New York, ISBN 0-387-94416-8.

Deimling, K. 1985. Nonlinear functional analysis, Springer-Verlag, Berlin, ISBN 3-540-13928-1.

Fiedler, B. and C. Rocha. 1996. Heteroclinic orbits of semilinear parabolic equations, J. Differential Equations 125, 239-281.

.1999. Realization of meander permutations by boundary value problems, J. Differential Equations 156, 282-308.

2000. Orbit equivalence of global attractors of semilinear parabolic differential equations, Trans. Amer. Math. Soc. 352, 257-284.

Fusco, G. and C. Rocha. 1991. A permutation related to the dynamics of a scalar parabolic PDE, J. Differential Equations 91, 111-137.

Gobbino, M. and M. Sardella. 1997. On the connectedness of attractors for dynamical systems, J. Differential Equations 133, 1-14.

Gottschalk, W. H. and G. A. Hedlund. 1955. Topological dynamics, American Mathematical Society Colloquium Publications, Vol. 36, American Mathematical Society, Providence, R. I.

Greiner, G. and M. Schwarz. 1991. Weak spectral mapping theorems for functional-differential equations, J. Differential Equations 94, 205-216.

Hale, J. K. 1965. Sufficient conditions for stability and instability of autonomous functionaldifferential equations, J. Differential Equations 1, 452-482.

.1970. A class of neutral equations with the fixed point property, Proc. Nat. Acad. Sci. U.S.A. 67, 136-137.

. 1981. Topics in dynamic bifurcation theory, CBMS Regional Conference Series in Mathematics, vol. 47, Conference Board of the Mathematical Sciences, Washington, D.C., ISBN 0-82181698-5.

1977. Theory of functional differential equations, Springer-Verlag, New York.

1983. Infinite-dimensional dynamical systems, Geometric Dynamics (Rio de Janeiro, 1981), Lecture Notes in Math., vol. 1007, Springer, Berlin, pp. 379-400.

. 1987. Asymptotic behavior of gradient dissipative systems, Dynamics of Infinite-Dimensional Systems (Lisbon, 1986), NATO Adv. Sci. Inst. Ser. F Comput. Systems Sci., vol. 37, Springer, Berlin, pp. 123-128.

1997. Dynamics of a scalar parabolic equation, Canad. Appl. Math. Quart. 5, 209-305.

. 2000. Dissipation and attractors, International Conference on Differential Equations, Vol 1, 2 (Berlin, 1999), World Sci. Publishing, River Edge, NJ, pp. 622-637.

Hale, J. K., J. P. LaSalle, and M. Slemrod. 1972. Theory of a general class of dissipative processes, J. Math. Anal. Appl. 39, 177-191.

Hale, J. K. and O. Lopes. 1973. Fixed point theorems and dissipative processes, J. Differential Equations 13, 391-402.

Hale, J. K. and K. R. Meyer. 1967. A class of functional equations of neutral type, Memoirs of the American Mathematical Society, No. 76, American Mathematical Society, Providence, R.I.

Hale, J. K., L. T. Magalhães, and W. M. Oliva. 2002. Dynamics in infinite dimensions, Applied Mathematical Sciences, vol. 47, Springer-Verlag, New York, ISBN 0-387-95463-5. 
Hale, J. K. and G. Raugel. 1989. Lower semicontinuity of attractors of gradient systems and applications, Ann. Mat. Pura Appl. (4) 154, 281-326.

1992. Convergence in gradient-like systems with applications to PDE, Z. Angew. Math. Phys. 43, 63-124.

1993. Attractors for dissipative evolutionary equations, International Conference on Differential Equations, Vol. 1, 2 (Barcelona, 1991), World Sci. Publishing, River Edge, NJ, pp. 322 .

2003. Regularity, determining modes and Galerkin methods, J. Math. Pures Appl. (9) 82 $1075-1136$.

2003. Infinite dimensional gradient systems (in preparation).

Hale, J. K. and S. M. Verduyn Lunel. 1993. Introduction to functional-differential equations, Applied Mathematical Sciences, vol. 99, Springer-Verlag, New York, ISBN 0-387-94076-6.

. 2002. Strong stabilization of neutral functional differential equations, IMA J. Math. Control Inform. 19, 5-23.

Hale, J. K. and K. P. Rybakowski. 1982. On a gradient-like integro-differential equation, Proc. Roy. Soc. Edinburgh Sect. A 92, 77-85.

Hale, J. K. and J. Scheurle. 1985. Smoothness of bounded solutions of nonlinear evolution equations, J. Differential Equations 56, 142-163.

Haraux, A. 1985. Two remarks on hyperbolic dissipative problems, Nonlinear Partial Differential Equations and Their Applications. Collège de France Seminar, Vol. VII (Paris, 1983-1984), Res. Notes in Math., vol. 122, Pitman, Boston, MA, pp. 6, 161-179.

1989. Une remarque sur la stabilisation de certains systèmes du deuxième ordre en temps, Portugal. Math. 46, 245-258. (French, with English summary)

Haraux, A. and M. A. Jendoubi. 1999. Convergence of bounded weak solutions of the wave equation with dissipation and analytic nonlinearity, Calc. Var. Partial Differential Equations 9, 95-124.

Henry, D. B. 1981. Geometric theory of semilinear parabolic equations, Lecture Notes in Mathematics, vol. 840, Springer-Verlag, Berlin, ISBN 3-540-10557-3.

. 1974. Linear autonomous neutral functional differential equations, J. Differential Equations 15, 106-128.

1985. Some infinite-dimensional Morse-Smale systems defined by parabolic partial differential equations, J. Differential Equations 59, 165-205.

1987. Topics in analysis, Publ. Sec. Mat. Univ. Autònoma Barcelona 31, 29-84.

1987. Generic properties of equilibrium solutions by perturbation of the boundary, Dynamics of Infinite-Dimensional Systems (Lisbon, 1986), NATO Adv. Sci. Inst. Ser. F Comput. Systems Sci., vol. 37, Springer, Berlin, pp. 129-139.

Hirsch, M. W. 1988. Stability and convergence in strongly monotone dynamical systems, J. Reine Angew. Math. 383, 1-53.

Hurley, M. 1991. Chain recurrence and attraction in noncompact spaces, Ergodic Theory Dynam. Systems 11, 709-729.

.1992. Noncompact chain recurrence and attraction, Proc. Amer. Math. Soc. 115, 1139-1148.

. 1995. Chain recurrence, semiflows, and gradients, J. Dynam. Differential Equations 7, 437456.

Iwasaki, N. 1969. Local decay of solutions for symmetric hyperbolic systems with dissipative and coercive boundary conditions in exterior domains, Publ. Res. Inst. Math. Sci. 5, 193-218. 
Kolmanovski1̌, V. and A. Myshkis. 1992. Applied theory of functional-differential equations, Mathematics and its Applications (Soviet Series), vol. 85, Kluwer Academic Publishers Group, Dordrecht, ISBN 0-7923-2013-1.

Kostin, I. N. 1990. A regular approach to a problem on the attractors of singularly perturbed equations, Zap. Nauchn. Sem. Leningrad. Otdel. Mat. Inst. Steklov. (LOMI) 181, 93-131, 187. (Russian, with English summary)

Ladyženskaja, O. A. 1972. The dynamical system that is generated by the Navier-Stokes equations, Zap. Naučn. Sem. Leningrad. Otdel. Mat. Inst. Steklov. (LOMI) 27, 91-115. (Russian)

.1987. On the determinination of minimal global attractors for the Navier-Stokes and other partial differential equations, Russian Mathematical Surveys 42, 27-73.

1991. Attractors for Semigroups and Evolution Equations.

LaSalle, J. P. 1976. The stability of dynamical systems, Society for Industrial and Applied Mathematics, Philadelphia, Pa.

Levin, J. J. and J. A. Nohel. 1964. On a nonlinear delay equation, J. Math. Anal. Appl. 8, 31-44.

Levinson, N. 1944. Transformation theory of non-linear differential equations of the second order, Ann. of Math. (2) 45, 723-737.

Mallet-Paret, J. 1976. Negatively invariant sets of compact maps and an extension of a theorem of Cartwright, J. Differential Equations 22, 331-348.

Mañé, R. 1981. On the dimension of the compact invariant sets of certain nonlinear maps, Dynamical Systems and Turbulence, Warwick 1980 (Coventry, 1979/1980), Lecture Notes in Math., vol. 898, Springer, Berlin, pp. 230-242.

Massatt, P. 1981. Stability and fixed points of point-dissipative systems, J. Differential Equations 40, 217-231.

1983. Limiting behavior for strongly damped nonlinear wave equations, J. Differential Equations 48, 334-349.

Matano, H. 1982. Nonincrease of the lap-number of a solution for a one-dimensional semilinear parabolic equation, J. Fac. Sci. Univ. Tokyo Sect. IA Math. 29, 401-441.

Nickel, K. 1962. Gestaltaussagen über Lösungen parabolischer Differentialgleichungen, J. Reine Angew. Math. 211, 78-94. (1 insert).

Nussbaum, R. D. 1972. Some asymptotic fixed point theorems, Trans. Amer. Math. Soc. 171, 349375 .

1973. Periodic solutions of analytic functional differential equations are analytic, Michigan Math. J. 20, 249-255.

Palis, J., Jr. and W. de Melo. 1982. Geometric theory of dynamical systems, Springer-Verlag, New York, ISBN 0-387-90668-1.

Pliss, V. A. 1966. Nonlocal problems of the theory of oscillations, Translated from the Russian by Scripta Technica, Inc. Translation edited by Harry Herman, Academic Press, New York.

Poláčik, P. 1991. Complicated dynamics in scalar semilinear parabolic equations in higher space dimension, J. Differential Equations 89, 244-271.

1995. High-dimensional $\omega$-limit sets and chaos in scalar parabolic equations, J. Differential Equations 119, 24-53.

. 1999. Persistent saddle connections in a class of reaction-diffusion equations, J. Differential Equations 156, 182-210.

. 2002. Parabolic equations: asymptotic behavior and dynamics on invariant manifolds, Handbook of Dynamical Systems, Vol. 2, North-Holland, Amsterdam, pp. 835-883. 
Poláčik, P. and K. P. Rybakowski. 1995. Imbedding vector fields in scalar parabolic Dirichlet BVPs, Ann. Scuola Norm. Sup. Pisa Cl. Sci. (4) 22, 737-749.

. 1996. Nonconvergent bounded trajectories in semilinear heat equations, J. Differential Equations 124, 472-494.

Prizzi, M. 1998. Realizing vector fields without loss of derivatives, Ann. Scuola Norm. Sup. Pisa Cl. Sci. (4) 27, 289-307 (1999).

Raugel, G. 2002. Global attractors in partial differential equations, Handbook of Dynamical Systems, Vol. 2, North-Holland, Amsterdam, pp. 885-982.

Sell, G. R. and Y. You. 2002. Dynamics of evolutionary equations, Applied Mathematical Sciences, vol. 143, Springer-Verlag, New York, ISBN 0-387-98347-3.

Simon, L. 1983. Asymptotics for a class of nonlinear evolution equations, with applications to geometric problems, Ann. of Math. (2) 118, 525-571.

Smale, S. 1967. Differentiable dynamical systems, Bull. Amer. Math. Soc. 73, 747-817.

Smith, H. L. and H. R. Thieme. 1991. Convergence for strongly order-preserving semiflows, SIAM J. Math. Anal. 22, 1081-1101.

Smoller, J. and A. Wasserman. 1984. Generic bifurcation of steady-state solutions, J. Differential Equations 52, 432-438.

Sternberg, N. 1990. One-to-oneness of the solution map in retarded functional-differential equations, J. Differential Equations 85, 201-213.

Temam, R. 1997. Infinite-dimensional dynamical systems in mechanics and physics, 2 nd ed., Applied Mathematical Sciences, vol. 68, Springer-Verlag, New York, ISBN 0-387-94866-X.

van Neerven, J. 1996. The asymptotic behaviour of semigroups of linear operators, Operator Theory: Advances and Applications, vol. 88, Birkhäuser Verlag, Basel, ISBN 3-7643-5455-0.

Yoshizawa, T. 1966. Stability theory by Liapunov's second method, Publications of the Mathematical Society of Japan, No. 9, The Mathematical Society of Japan, Tokyo.

Zelenjak, T. I. 1968. Stabilization of solutions of boundary value problems for a second-order parabolic equation with one space variable, Differencial'nye Uravnenija 4, 34-45. (Russian) 